\title{
Kiloparsec-scale Simulations of Star Formation in Disk Galaxies. IV. Regulation of Galactic Star Formation Rates by Stellar Feedback
}

\author{
Michael J. Butler ${ }^{1}$, Jonathan C. Tan ${ }^{2,3}$, Romain Teyssier ${ }^{4}$, Joakim Rosdahl ${ }^{5,6}$, Sven Van Loo ${ }^{7}$, and Sarah Nickerson ${ }^{4}$ \\ ${ }^{1}$ Max Planck Institute for Astronomy, Königstuhl 17, D-69117 Heidelberg, Germany \\ 2 Departments of Astronomy \& Physics, University of Florida, Gainesville, FL 32611, USA \\ National Astronomical Observatory, Mitaka, Tokyo 181-8588, Japan \\ ${ }^{4}$ Institute for Computational Science, University of Zurich, 8049 Zurich, Switzerland \\ ${ }^{5}$ Leiden Observatory, Leiden University, P.O. Box 9513, NL-2300 RA Leiden, the Netherlands \\ ${ }^{6}$ Univ Lyon, Univ Lyon1, Ens de Lyon, CNRS, Centre de Recherche Astrophysique de Lyon UMR5574, F-69230, Saint-Genis-Laval, France \\ ${ }^{7}$ School of Physics and Astronomy, University of Leeds, Leeds LS2 9JT, UK \\ Received 2017 March 11; revised 2017 April 27; accepted 2017 April 27; published 2017 May 26
}

\begin{abstract}
Star formation from the interstellar medium of galactic disks is a basic process controlling the evolution of galaxies. Understanding the star formation rate (SFR) in a local patch of a disk with a given gas mass is thus an important challenge for theoretical models. Here we simulate a kiloparsec region of a disk, following the evolution of self-gravitating molecular clouds down to subparsec scales, as they form stars that then inject feedback energy by dissociating and ionizing UV photons and supernova explosions. We assess the relative importance of each feedback mechanism. We find that $\mathrm{H}_{2}$-dissociating feedback results in the largest absolute reduction in star formation compared to the run with no feedback. Subsequently adding photoionization feedback produces a more modest reduction. Our fiducial models that combine all three feedback mechanisms yield, without fine-tuning, SFRs that are in excellent agreement with observations, with $\mathrm{H}_{2}$-dissociating photons playing a crucial role. Models that only include supernova feedback - a common method in galaxy evolution simulations-settle to similar SFRs, but with very different temperatures and chemical states of the gas, and with very different spatial distributions of young stars.
\end{abstract}

Key words: evolution - galaxies: ISM - galaxies: star formation - ISM: clouds - radiative transfer - stars: formation

\section{Introduction}

The formation of stars from the interstellar medium (ISM) of galactic disks is one of the basic processes that controls the evolution of galaxies. Most stellar populations are built up in this way, including those in typical Milky Way-like disk galaxies, as well as those formed in starbursts due to galactic interactions that drive large quantities of the ISM gas to more compact circumnuclear disks. Understanding the star formation rate (SFR) per unit area, $\Sigma_{\mathrm{SFR}}$, from a given galactic disk system as characterized by its total gas mass per unit area, $\Sigma_{g}$, is thus a basic challenge for theoretical models of star formation and galaxy evolution. Empirically, there is a well-established correlation of $\Sigma_{\mathrm{SFR}}=(6.3 \pm 1.8) \times 10^{-3}$ $\left(\Sigma_{g} / 10 M_{\odot} \mathrm{pc}^{-2}\right)^{1.4 \pm 0.15} M_{\odot} \mathrm{yr}^{-1} \mathrm{kpc}^{-2}$ based on global averages of disk systems (Kennicutt 1998). Considering only the mass surface density of molecular gas, $\Sigma_{\mathrm{H} 2}$, and averaging on smaller $\sim \mathrm{kpc}$ scales, a linear relation $\Sigma_{\mathrm{SFR}}=(5.3 \pm 0.3) \times$ $10^{-3}\left(\Sigma_{\mathrm{H} 2} / 10 M_{\odot} \mathrm{pc}^{-2}\right) M_{\odot} \mathrm{yr}^{-1} \mathrm{kpc}^{-2}$ has been derived (Bigiel et al. 2008; Leroy et al. 2008). These SFRs are of relatively low efficiency: only about $4 \%$ percent of the total gas content in a given annulus is turned into stars per local orbital time (e.g., Leroy et al. 2008; Tan 2010). Zooming in to the scales where stars actually form, the majority are born in very localized, $\sim$ parsec-scale clumps, i.e., protostar clusters, within turbulent, magnetized giant molecular clouds (GMCs), which are themselves $\sim 10$ to $100 \mathrm{pc}$ in size (McKee \& Ostriker 2007). The inefficiency of star formation continues down to these scales: only a few percent of GMC and clump gas forms stars per local free-fall time (Krumholz \& Tan 2007; Da Rio et al. 2014).
The physics of the star formation process is expected to involve a competition between gravitational collapse and various forms of pressure support in the GMCs and their clumps (e.g., thermal, turbulent, magnetic, radiation). However, thermal pressure is relatively unimportant at the $\lesssim 20 \mathrm{~K}$ temperatures of most GMC material. The inefficiency of observed SFRs in GMCs implies that some combination of turbulence, magnetic fields, and feedback is playing a crucial role in regulating SFRs, making the rates much smaller than those that would result from unsupported, free-fall collapse of the clouds. However, the relative importance of these inhibiting factors remains very uncertain. On the theoretical side, this is because numerical simulations of the ISM and star formation are challenging due to the large range of scales that must be followed, the uncertain choices that need to be made when specifying initial conditions, and the wide variety of physical processes that must be considered.

In this paper, we present numerical simulations that explore star formation in galactic disks, focusing on the role of feedback in regulating the ISM and star formation activity. This is the fourth in a series of papers describing $3 \mathrm{D}$ simulations of a $1 \mathrm{kpc}^{2}$ patch of a galactic disk extracted from the global galaxy simulation of Tasker \& Tan (2009). In Van Loo et al. (2013, Paper I), we followed the patch for $10 \mathrm{Myr}$ down to a resolution of $0.5 \mathrm{pc}$. Star formation was included, with star particles with a minimum mass of $100 M_{\odot}$ forming in gas above a density threshold of $n_{\mathrm{H}, *}>10^{5} \mathrm{~cm}^{-3}$ at a star formation efficiency per local free-fall time of $\epsilon_{\mathrm{ff}}=0.02$. No feedback processes aside from a constant FUV field (of strength $4 G_{0}$, where $1 G_{0}$ is the standard Habing (1968) intensity) were included, and after 
10 Myr we observed SFR surface densities $\sim 100$ times higher than those in galaxies of comparable gas mass surface density (e.g., Bigiel et al. 2008). In Paper I, we speculated that this was due to a lack of magnetic fields and/or local feedback from young stars.

In Van Loo et al. (2015, hereafter Paper II) we explored the effects of magnetic fields of varying strength on the same kiloparsec-scale patch of the Tasker \& Tan (2009) galactic disk. Here we found that magnetic fields suppressed the overall SFRs by up to a factor of two; however, this result was strongly influenced by the presence of a magnetically supercritical starburst region. In other regions, larger suppression rates were observed.

In Butler et al. (2015, hereafter Paper III), we followed the kiloparsec-scale patch down to much higher resolution $(\sim 0.1 \mathrm{pc})$ for $4 \mathrm{Myr}$. Star formation was included with the same recipe as in Papers I and II, but now with a higher density threshold of $n_{\mathrm{H}, *}>10^{6} \mathrm{~cm}^{-3}$ and a minimum star particle mass of $10 M_{\odot}$. As in Paper I, we did not include magnetic fields or feedback from star particles. Enabled by our higher resolution, we explored the structural, kinematic, and dynamical properties of the large filaments and clumps and compared them, when possible, to observations of Infrared Dark Clouds (IRDCs) and long molecular filaments that have been identified in the Galactic plane. We found that by many metrics, including too high dense $\left(\Sigma>1.0 \mathrm{~g} \mathrm{~cm}^{-2}\right)$ gas mass fractions, too high mass per unit length dispersion and velocity gradients along the filaments, and too high velocity dispersion for a given mass per unit length, our simulated filaments and clumps differ greatly compared to observed clouds. We therefore concluded that IRDCs do not form from the global fast collapse of GMCs, but rather we expect them to be strongly influenced by dynamically important magnetic fields.

The important features that distinguish the simulations of this current paper (Paper IV) from previous works are (1) the inclusion of dissociating and ionizing feedback, in combination with supernova feedback; (2) the adopted simulation setup that is inherited from a shearing, global disk simulation; (3) the high dynamic range from $1 \mathrm{kpc}$ down to $0.5 \mathrm{pc}$; (4) the high, realistic density threshold for initiating star formation of $n_{\mathrm{H}, *}>10^{5} \mathrm{~cm}^{-3}$ and the requirement that this gas be mostly molecular; and (5) the use of a star formation efficiency per local free-fall time of $2 \%$. These features of the star formation subgrid model are motivated by observational studies of star-forming molecular clouds (e.g., Zuckerman \& Evans 1974; Krumholz \& Tan 2007; Da Rio et al. 2014; Tan et al. 2014). They are an approximate attempt to account for the regulation of SFRs by a combination of turbulence and magnetic fields operating on subgrid scales.

In comparison, Walch et al. (2015) presented simulations of a 500 pc-sized patch of a non-shearing disk with fiducial resolution of about $4 \mathrm{pc}$, studying only localized feedback from supernovae, although in a background UV radiation field. Kim et al. (2013) simulated a shearing 512 pc-sized patch of disk down to $2 \mathrm{pc}$ resolution to study star formation from "dense" gas $\left(n_{\mathrm{H}, *}>200 \mathrm{~cm}^{-3}\right)$ and subsequent momentum injection from SN feedback. Kim \& Ostriker (2015) included the effects of magnetic fields, finding only modest reductions in SFRs of 25\%. On larger scales, Hopkins et al. (2012) have presented smooth particle hydrodynamics (SPH) simulations of galaxies with up to subparsec resolution in which stars form when $n_{\mathrm{H}, *}>1000 \mathrm{~cm}^{-3}$ and including momentum feedback representing stellar winds, radiation pressure and supernovae, and an approximate Strömgren-type model for ionization feedback. However, the SPH method has been criticized for being very diffusive and for having difficulties in accurately modeling the multiphase ISM that is expected in regions affected by strong feedback (Agertz et al. 2007). Agertz \& Kratsov (2015) have simulated a global disk galaxy with a cosmological zoom-in grid-based adaptive mesh refinement (AMR) simulation that includes energy and momentum feedback from winds, radiation pressure, and supernova feedback, but with a resolution of only $75 \mathrm{pc}$. The treatment of radiative transfer in the above simulations has generally been very approximate in comparison to the moment-based method we utilize here. Rosdahl et al. (2015) have presented global galaxy simulations including photoionization, radiation pressure, and supernova feedback, but with minimum resolutions of about $20 \mathrm{pc}$, which is still too small to resolve GMCs. Photodissociation feedback with a moment-based method of radiative transfer has not been included in any of the above simulations.

We note that after the submission of the first version of our paper, the study of Peters et al. (2017) was published, which carried out similar simulations on a $0.5 \mathrm{kpc}$ by $0.5 \mathrm{kpc}$ nonshearing disk patch at $4 \mathrm{pc}$ resolution that model star formation with a sink particle method and include dissociating feedback, in addition to photoionization, stellar winds, and supernovae. One of the main conclusions of the Peters et al. study is that FUV-dissociating feedback is needed to produce SFRs, i.e., gas depletion times, in agreement with observed galactic disks.

\section{Methods}

Our approach is to focus on a kiloparsec-sized patch of a typical galactic disk, extended vertically also by a kiloparsec (i.e., to $500 \mathrm{pc}$ on either side of the disk midplane), with a flat, i.e., constant, rotation curve of $200 \mathrm{~km} \mathrm{~s}^{-1}$ circular velocity. The center of the patch is at a galactocentric radius of $4.25 \mathrm{kpc}$. This kiloparsec-sized region is large enough to be sensitive to the main effects of galactic dynamics, including the shear flow due to differential rotation and the vertical structure of the disk. At the same time, the region is small enough that it is practical to have resolution down to $0.5 \mathrm{pc}$ scales that can resolve GMCs. For initial conditions, following Papers I, II, and III, we adopt a structured ISM with average gas content of $\Sigma_{g}=17 M_{\odot} \mathrm{pc}^{-2}$ that was extracted from a lower, $8 \mathrm{pc}$, resolution global galaxy simulation (Tasker \& Tan 2009 (TT09)), which already formed a population of self-gravitating GMCs evolved to the point of quasistatistical equilibrium via shear-driven collisions and interactions. These initial conditions are shown in the left columns of Figures 1 and 2, which display top-down and in-plane views of the simulation volume. We note that the TT09 simulation did not include star formation or feedback and so the initial conditions here have only been sculpted by the processes of gravitational instability and cloud collisions in a shearing disk. As discussed in TT09 and Paper I, the GMCs have properties such as masses and virial parameters that are quite similar to observed GMCs, but with one main difference: they have mass surface densities about a factor of two greater than local Milky Way GMCs. 

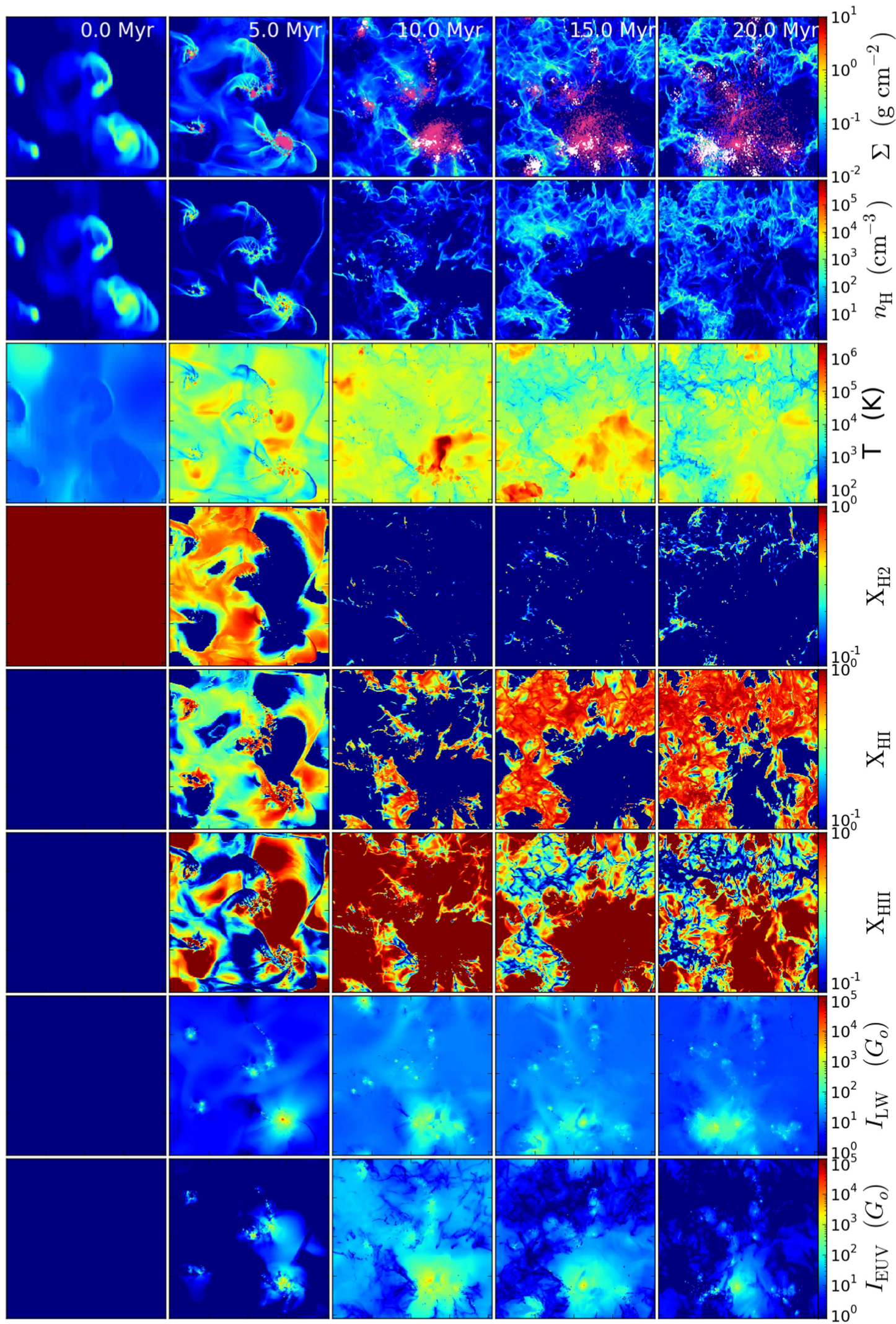

Figure 1. Evolution of the galactic interstellar medium for the fiducial simulation run that includes dissociating, ionizing, and supernova feedback (Run LW+EUV $+\mathrm{SN}$ ), as viewed projected along the $z$-axis from above the disk (i.e., a top-down view, with each panel showing the full $1 \mathrm{kpc}$-sided disk patch, with box center at a $4.25 \mathrm{kpc}$ distance from the galactic center, which is located to the left) at times $0,5,10,15$, and $20 \mathrm{Myr}$ (left to right columns). Top row: total gas mass surface density, $\Sigma_{g}$, along with young stars formed before $5 \mathrm{Myr}$ (red points) and after $5 \mathrm{Myr}$ (white points). Second row: mass-weighted hydrogen number density $n_{\mathrm{H}}$. Third row: mass-weighted temperature, $T$. Fourth, fifth, and sixth rows: mass-weighted molecular hydrogen fraction, $X_{\mathrm{H} 2}$, atomic hydrogen fraction, $X_{\mathrm{HI}}$, and ionized hydrogen fraction, $X_{\mathrm{H} \text { II }}$. Seventh row: mass-weighted Lyman-Werner (i.e., $\mathrm{H}_{2}$ dissociating) radiation intensity $I_{\mathrm{LW}}$, which tends to emphasize the radiation field that is having the main destructive effect on dense gas clouds. Bottom row: mass-weighted hydrogen ionizing intensity $I_{\mathrm{EUv}}$. 


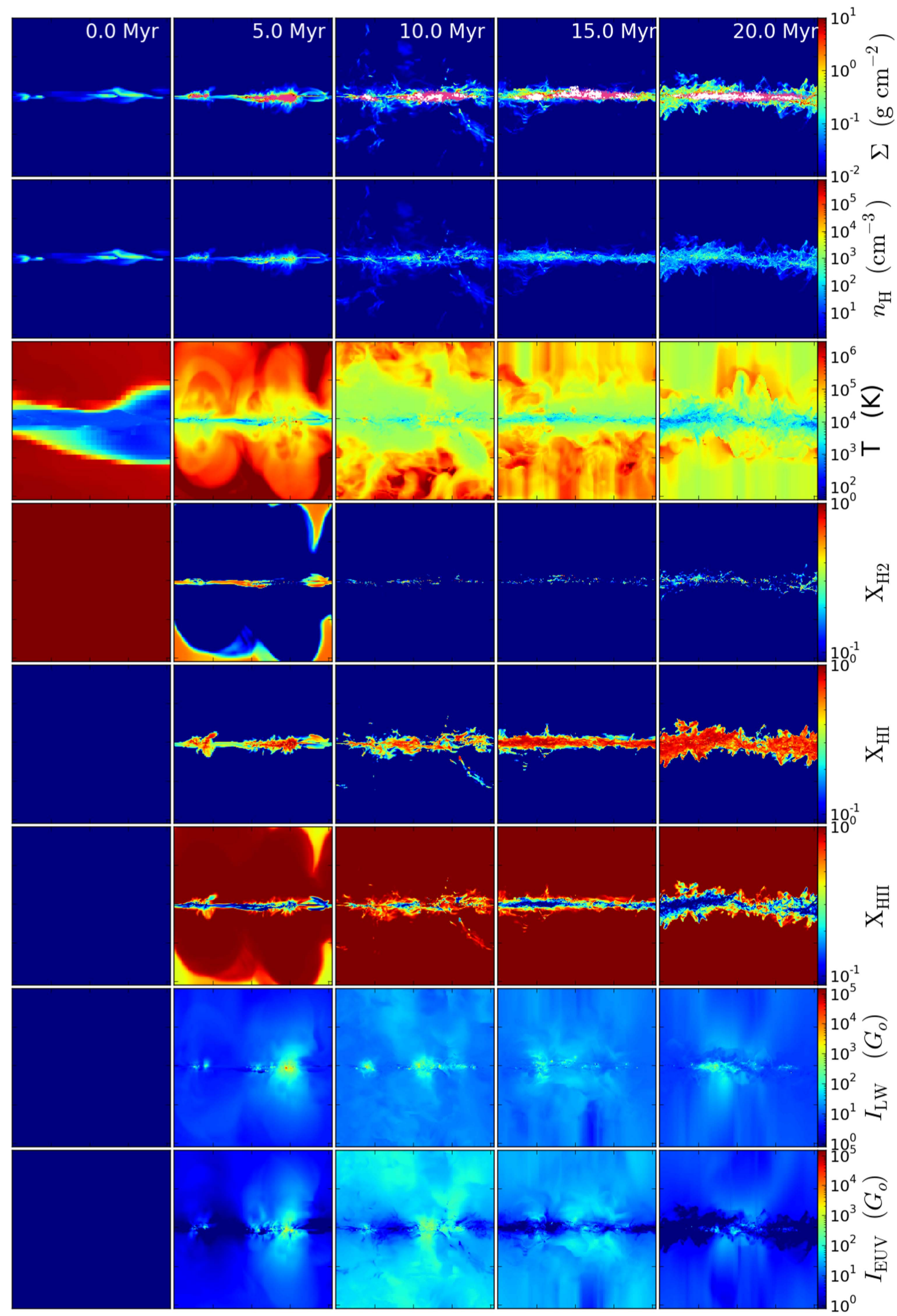

Figure 2. As in Figure 1, but projected along the $y$-axis, i.e., an in-plane view, for Run LW+EUV+SN. 
Partly for this reason, when these initial conditions are adopted in models that do include star formation and feedback, there is an initial transient burst of star formation that we regard as unrealistic. Thus, to mitigate its effects, we carry out evolution with an "initial feedback model," discussed below, in a first phase from 0 to $5 \mathrm{Myr}$, and then adopt the conditions at $5 \mathrm{Myr}$ as our starting point for investigating the different effects of feedback implementation.

We also note that our simulation setup, again following Papers I, II, and III, is not that of a shearing box, but rather is transformed into the rest frame of the center of the box at a galactocentric distance of $4.25 \mathrm{kpc}$. The gas in the disk patch inherits shearing motions that were present in the global disk (and GMCs also inherit a random velocity dispersion of $\sim 10 \mathrm{~km} \mathrm{~s}^{-1}$ ), but these are not maintained during our simulation. For this reason, we limit the evolution to a period of $20 \mathrm{Myr}$, i.e., about one flow crossing time at the maximum shear velocity. The boundary conditions of the volume are set to be outflow in the vertical directions and periodic in the disk plane.

Using the AMR radiation-hydrodynamics code RAMSES-RT (Rosdahl et al. 2013), we have included several physical processes in the simulations, especially heating and cooling of molecular, atomic, and ionized gas; star formation from dense $\left(n_{\mathrm{H}}>10^{5} \mathrm{~cm}^{-3}\right)$, molecular $\left(X_{\mathrm{H} 2}>0.9\right)$ gas, i.e., the creation of "star particles" that each represents a small cluster of stars of $100 M_{\odot}$, with average feedback properties assessed by assuming a standard Chabrier (2003) stellar initial mass function (IMF); and several feedback mechanisms from these newly formed stars. In particular, we explore the effects of three types of stellar feedback: $\mathrm{H}_{2}$ dissociation by Lyman-Werner (LW) band ultraviolet photons, photoionzation of hydrogen by extreme ultraviolet (EUV) photons that have $>13.6 \mathrm{eV}$ energies, and supernovae, i.e., the explosive death of massive stars that occurs $3 \mathrm{Myr}$ after their formation, leading to the release of $10^{51} \mathrm{erg}$ of kinetic energy. The radiation from the stars is propagated on the AMR grid using a first-order Godunov method, using the moment-based M1 approximation for the Eddington Tensor. The primary advantage of this method is that it is independent of the number of radiation sources, allowing us to study the effects of feedback from many thousands of star particles.

As discussed above, the same initial conditions have also been simulated previously with two other AMR simulation codes: Enzo (Bryan et al. 2014), for the case of pure hydrodynamics with no direct stellar feedback, but including the effect of a diffuse background FUV radiation field (Van Loo et al. 2013; Butler et al. 2015); and $M G$ (Falle et al. 2012) for the case of magnetohydrodynamics (MHD), but with no direct feedback (Van Loo et al. 2015). Thus, we are able to compare our results with those of these previous simulations to examine the relative importance of magnetic fields and feedback.

The simulations contain four levels of AMR on top of the root grid resolution of $7.8 \mathrm{pc}$ (i.e., $128^{3}$ over the $\mathrm{kpc}^{3}$ volume), reaching a minimum cell size of $0.49 \mathrm{pc}$ at the finest level (i.e., equivalent to the resolution of a $2048^{3}$ uniform grid simulation). Cells are refined when the total mass within exceeds $800 M_{\odot}$. For example, on refinement to the finest level, this corresponds to a density in a 1 pc-sized cell of $n_{\mathrm{H}}=2.3 \times 10^{4} \mathrm{~cm}^{-3}$, which is insufficient to resolve the Jeans length for temperatures that may be as low as $\sim 10 \mathrm{~K}$.
As with previous papers in this series, the primary goal is not to be able to properly resolve the details of gravitational fragmentation in any particular GMC, but rather to have an approximate estimate of the locations and mass fractions of gas that reach densities of $\sim 10^{5} \mathrm{~cm}^{-3}$ that are necessary for star formation.

We evolve the simulations for $20 \mathrm{Myr}$, first with an "initial feedback model" until $5 \mathrm{Myr}$, and then with a variety of different feedback implementations from 5 to $20 \mathrm{Myr}$. As described below, the goal of the initial feedback model, which has reduced FUV feedback, is to mitigate the effects of the initial transient burst of star formation that depends on adopted initial conditions.

In the following subsections, we describe the main physical processes that have been included in the simulation code.

\subsection{Star Formation}

We utilize a subgrid model for star formation in which gas is converted, stochastically, into star particles at a fixed efficiency $\epsilon_{\mathrm{ff}}=0.02$ per free-fall time, if a cell exceeds a given threshold density, $n_{\mathrm{H}, *}=10^{5} \mathrm{~cm}^{-3}$, and molecular hydrogen mass fraction of $X_{\mathrm{H} 2, *}=0.9$. This choice of $\epsilon_{\mathrm{ff}}$ is motivated by the empirical results of Krumholz \& Tan (2007) and is the same value used in the simulations of Van Loo et al. $(2015,2013)$. The choice of $n_{\mathrm{H}, *}$ is also motivated by observations of local star-forming clouds, especially IRDCs, which can achieve these densities, while remaining mostly starless (Butler \& Tan 2012; Tan et al. 2014). The choice of $n_{\mathrm{H}, *}$ is also the same as that used in the simulations of Van Loo et al. $(2013,2015)$. The value of $X_{\mathrm{H} 2, *}$ is motivated by the fact that, empirically, star formation is seen to occur exclusively in molecular clouds. Physically, the reason for this may be that this allows cooling to low temperatures of $\sim 10 \mathrm{~K}$, helped by $\mathrm{CO}$ line cooling, which then allows dense, supersonically turbulent gas clumps to form. Their relatively low ionization fractions and turbulent conditions may be crucial for mediating the loss of magnetic flux support from the gas, e.g., by turbulent reconnection (Lazarian \& Vishniac 1999; Eyink et al. 2011).

Star formation is then modeled by the creation of "star particles" that are intended to represent a small cluster of young stars. On their creation, the equivalent mass is removed instantaneously from the gas in the relevant cell. The star particles only feel gravitational forces from the surrounding gas and the background galactic potential. Also, they do not accrete any material once formed. We adopt a minimum star particle mass of $100 M_{\odot}$, with this choice being motivated by the minimum gas mass that is present in a starforming $0.5 \mathrm{pc}$-sized cell of $400 M_{\odot}$, given the choice of $n_{\mathrm{H}, *}=10^{5} \mathrm{~cm}^{-3}$. In typical simulation time steps, the mass of new young stars that should form is much less than $100 M_{\odot}$, so a star particle is only formed with a probability that yields an overall, time-averaged SFR equal to that set by the choice of $\epsilon_{\mathrm{ff}}=0.02$. Thus, all star particles in the simulations are in fact born with masses of $100 M_{\odot}$, and when they are formed, the mass of gas in the natal cell undergoes at most a $\sim 25 \%$ change. 
Table 1

Radiation Group Energy Intervals and Properties ${ }^{\mathrm{a}}$

\begin{tabular}{|c|c|c|c|c|c|c|}
\hline Photon Group & $\begin{array}{c}\epsilon_{0} \\
(\mathrm{eV})\end{array}$ & $\begin{array}{c}\epsilon_{1} \\
(\mathrm{eV})\end{array}$ & $\begin{array}{c}\sigma_{\mathrm{H}_{2}} \\
\left(\mathrm{~cm}^{2}\right)\end{array}$ & $\begin{array}{c}\sigma_{\mathrm{H}} \\
\left(\mathrm{cm}^{2}\right)\end{array}$ & $\begin{array}{c}\sigma_{\mathrm{He}_{1}} \\
\left(\mathrm{~cm}^{2}\right)\end{array}$ & $\begin{array}{l}\sigma_{\mathrm{He} \mathrm{II}} \\
\left(\mathrm{cm}^{2}\right)\end{array}$ \\
\hline LW & 12.00 & 13.60 & $3.000 \times 10^{-22}$ & 0.000 & 0.000 & 0.000 \\
\hline $\mathrm{EUV}_{\mathrm{H} \mathrm{I}}$ & 13.60 & 24.59 & 0.000 & $3.007 \times 10^{-18}$ & 0.000 & 0.000 \\
\hline EUV $_{\mathrm{He} \text { I }}$ & 24.59 & 54.42 & 0.000 & $5.687 \times 10^{-19}$ & $4.478 \times 10^{-18}$ & 0.000 \\
\hline $\mathrm{EUV}_{\mathrm{He} \text { II }}$ & 54.42 & $\infty$ & 0.000 & $7.889 \times 10^{-20}$ & $1.197 \times 10^{-18}$ & $1.055 \times 10^{-18}$ \\
\hline
\end{tabular}

Note.

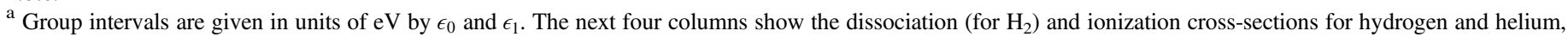

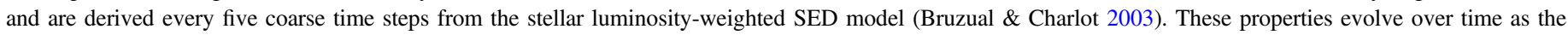
stellar populations age, varying by a few percent.

\subsection{Stellar Evolution and Feedback}

The star particles are then sources of feedback on the surrounding gas. The feedback properties are based on that expected from a stellar population with initial masses distributed according to the Chabrier (2003) IMF with upper and lower mass limits of $120 M_{\odot}$ and $0.1 M_{\odot}$. In reality, one expects stochastic sampling of the upper end of the IMF, but this effect is not included here. We anticipate that it would make only minor differences due to the large number of star particles that are formed in the simulations. Thus, we utilize IMF-averaged stellar evolutionary tracks from Bruzual \& Charlot (2003) that follow evolution from the zero-age main sequence to later stages (see Rosdahl et al. 2015 for more details).

In this study, our primary focus is to investigate the thermal and chemical feedback due to UV radiation. Thus, the FUV radiation field is constructed, which induces photoelectric heating feedback. It also leads to the dissociation of $\mathrm{H}_{2}$. The EUV radiation field leads to the ionization of $\mathrm{H}$, with the ionized gas heated to near $10^{4} \mathrm{~K}$. The elevated thermal pressure of these $\mathrm{H}$ II regions is another form of feedback. The radiative transfer methods necessary to model this feedback are described below, while the types of radiation feedback are summarized in Table 1.

Note that in this work, we have not modeled the effects of radiation pressure feedback, which is deferred to a future study (J. Rosdahl et al. 2017, in preparation). Nor have we modeled the momentum input from stellar wind feedback, which is difficult to resolve on the scales followed here, especially the effects of overlapping wind bubbles from individual stars, which will lead to a reduction in the overall momentum injection due to oppositely directed flows.

Supernova feedback is modeled with a single injection from each star particle (of total mass $m_{*}$ ) into its host cell $3 \mathrm{Myr}$ after the particle is formed of ejecta mass $m_{\mathrm{ej}}=\eta_{\mathrm{SN}} m_{*}$ and thermal energy $\epsilon_{\mathrm{SN}}=10 \eta_{\mathrm{SN}} \times 10^{51} \operatorname{erg}\left(m_{*} / 100 M_{\odot}\right)$. We choose a value of $\eta_{\mathrm{SN}}=0.1$, resulting in each star particle injecting the energy and mass expected from a single supernova progenitor star of initial mass of approximately $10-20 M_{\odot}$. It is worth noting that, thanks to our high mass resolution, we are effectively modeling each individual supernova explosion, one star at a time. Following the supernova event, cooling is delayed by a dissipation timescale $t_{\text {diss, }}$, which we have set to 50,000 years, given typical densities on the scales of our minimum resolution of $0.5 \mathrm{pc}$. In practice, this is implemented by turning off cooling in a cell (and adjacent cells) that contains a star particle that underwent a supernova explosion in the last 50,000 years (see Teyssier et al. 2013; Rosdahl et al. 2015).

To enable the rapid development of a realistic ISM structure that is approximately similar to that in the inner Milky Way disk, e.g., at $\sim 4 \mathrm{kpc}$ galactocentric radius, we first run the simulation from 0 to $5 \mathrm{Myr}$ with an "initial feedback model" that includes FUV, EUV, and SN feedback. However, because there is an initial transient burst of star formation that occurs before feedback can begin to fully regulate the ISM and because the FUV feedback from stars is quite long-lived (i.e., $\sim 10 \mathrm{Myr}$ ), we artificially lower the intensity of FUV radiation from these initial phase star particles by a reduction factor of 0.1 , while keeping EUV and $\mathrm{SN}$ feedback as normal. The net result of this model is that by $5 \mathrm{Myr}$, the FUV intensity from the initial phase stars is at a median level of $5.3 G_{0}$ within the $100 \mathrm{pc}$ thick-disk midplane region, where $1 G_{0}$ is the standard Habing (1968) intensity. This value is similar to that of the Milky Way at galactocentric radii of $\sim 4 \mathrm{kpc}$ (Wolfire et al. 2003). The main focus of our work is then to investigate how implementing different feedback methods from stars formed after $5 \mathrm{Myr}$ affects ISM and star formation properties from the period from 5 to $20 \mathrm{Myr}$. Note that the residual effects of feedback from the initial phase stars continue after $5 \mathrm{Myr}$, but quite rapidly lose importance due to the short timescales associated with massive star evolution.

\subsection{Radiative Transfer}

Details of the radiative transfer algorithm can be found in Rosdahl et al. (2013). Here we outline the main methods and approximations used in this paper. For stability reasons, the radiative transfer time step scales inversely with the speed of light $c$. To avoid very small time steps relative to those set by the hydrodynamics, we use the "reduced speed of light approximation" (Gnedin \& Abel 2001), and limit the propagation of radiation to a global speed, $\bar{c}_{\text {red }} \ll c$, where $c$ is the true speed of light. In our simulations, we adopt the value of $\bar{c}_{\text {red }}=0.005 c$, i.e., $1500 \mathrm{~km} \mathrm{~s}^{-1}$. We expect the effects of this choice to be relatively minor (see Rosdahl et al. 2015 for explorations of the effect of this choice on SFRs in larger-scale simulations). It is important to note that this approximation is only valid if the reduced speed of light $\bar{c}_{\text {red }}$ results in a light crossing time that is short compared to 
the smallest of the sound crossing time, the recombination time, and the advection time. We have checked a posteriori that our simulation indeed satisfies these requirements. For the transport of radiation, as described in detail in Rosdahl \& Teyssier (2015), we use a first-order moment method, which tracks in each cell the radiation energy density $E$ (zeroth moment, energy per unit volume) and the bulk radiation flux vector $\boldsymbol{F}$ (first moment, energy per unit area, and time). The system of moment equations is closed with the M1 expression for the radiation pressure tensor, which has the large numerical advantage that it can be evaluated purely from the local quantities $E$ and $\boldsymbol{F}$. A moment method treats the radiation as a fluid, rather than tracking individual rays, giving the very important advantage that we can treat a large number of radiation sources. As demonstrated in detail in Rosdahl \& Teyssier (2015), the sacrifice that comes with this is that rays from individual sources are treated in an average sense in every volume element, resulting in a radiation field that is not as accurate as with ray tracing, on scales of the source separation. In Rosdahl et al. (2015), we demonstrate that qualitatively we still retain a correct radiation field in a galaxy disk setup.

The two moments are the radiation energy density $E\left(\mathrm{erg} \mathrm{cm}^{-3}\right)$

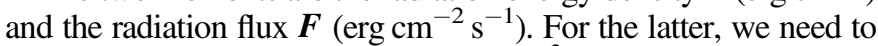
model the radiation pressure $\boldsymbol{P}\left(\mathrm{erg} \mathrm{cm}^{-3}\right)$, for which we use the M1 closure. The set of moment equations is solved separately for each radiation group, which is defined for a specific frequency range. For this work, we model four radiation groups (see Table 1) that determine the photodissociation of $\mathrm{H}_{2}$, the ionization of $\mathrm{HI}$, the ionization of $\mathrm{He} \mathrm{I}$, and the ionization of $\mathrm{He} \mathrm{II}$, respectively.

The ionization fractions are defined as $X_{\mathrm{H} \mathrm{II}}=n_{\mathrm{H} \mathrm{II}} / n_{\mathrm{H}}$, $X_{\mathrm{He} \text { II }}=n_{\mathrm{He} \text { II }} / n_{\mathrm{H}}$, and $X_{\mathrm{He} \mathrm{III}}=n_{\mathrm{He} \text { III }} / n_{\mathrm{H}}$. While previous work using Ramses-RT at much larger scales only considered the atomic and ionized species of hydrogen and helium (e.g., Rosdahl et al. 2015), with our higher resolution and denser, colder gas, it becomes important to model the formation and destruction of molecules. We introduce a photodissociating radiation model for molecular hydrogen that is included in the non-equilibrium chemistry solver. $\mathrm{H}_{2}$ is formed on dust grains as described by Draine \& Bertoldi (1996), as well as from the gas phase onto $\mathrm{H}^{-}$, assuming equilibrium abundances for $\mathrm{H}^{-}$ (see Abel et al. 1997), the latter of which is only important at low metallicities.

$\mathrm{H}_{2}$ is dissociated either through collisions with $\mathrm{H}$ I (Dove \& Mandy 1986; Abel et al. 1997) or through photodissociation by photons in the LW band. For the latter, we use the radiative transfer algorithm of RAMSES-RT, without considering Doppler effects and the associated relativistic corrections to the moment equations. In other words, this amounts to ignoring the effects of velocity gradients in the $\mathrm{H}_{2}$ opacity calculation, while still properly accounting for shielding of LW radiation due to the column density of $\mathrm{H}_{2}$ in dense molecular clouds. These effects are also ignored for the ionization of hydrogen and helium.

\subsection{Heating and Cooling}

Hydrogen and helium thermochemistry is calculated based on the absorption and emission of photons by hydrogen and helium species, photoelectric heating due to electrons ejected from dust grains, as well as equilibrium metal and dust cooling assuming solar metallicity. The gas thermal energy density is tracked in each cell along with the abundances of the species that interact with the photons. We utilize the same non-equilibrium thermochemical network described in Rosdahl et al. (2015). In this network, HI, He I, and He II interact with photons through photoionization, and $\mathrm{HII}$, He II, and He III through recombination. In this paper, we have additionally considered the cooling and heating contributions from the dissociation of molecular hydrogen $\mathrm{H}_{2}$ by photons in the LW band, as well as line emission from molecular rotational and vibrational levels and fine-structure atomic levels. We also include free-free, freebound, and two-photon cooling, as well as photoelectric heating via dust grains that scales with the LW flux as in Forbes et al. (2016).

While we model non-equilibrium hydrogen and helium thermochemistry, a non-equilibrium description of the thermochemistry of metal species is beyond the scope of this work. Instead, we use tabulated metal contributions to the cooling rates, including dust cooling, extracted from CLOUDY (Ferland et al. 2013), stored as a function of the local temperature, and assumed to scale linearly with the gas density and metallicity.

\section{Results}

\subsection{Effect of Feedback on Global Evolution of the ISM}

We follow the evolution of the ISM in a series of galactic disk patch simulations for $20 \mathrm{Myr}$. We will see that this is a long enough time for the results of different feedback mechanisms to become readily apparent and for the properties of the ISM, including its SFR, to reach a quasi-statistical equilibrium. As discussed in Section 2.2, to help enable the rapid development of a realistic ISM structure that is approximately similar to that in the inner Milky Way disk, e.g., at a distance of $\sim 4 \mathrm{kpc}$ from the Galactic, we first run the simulation from 0 to $5 \mathrm{Myr}$ with an "initial feedback model" that includes FUV (reduced by a factor of 0.1), EUV, and SN feedback. The net result of this model is that by 5 Myr, the FUV intensity from the "initial phase" stars is at a median level of $5.3 G_{0}$ within the $100 \mathrm{pc}$ thick-disk midplane region.

After $5 \mathrm{Myr}$, different cases of feedback are then investigated for the newly formed stars in a sequence of model runs: (1) no feedback, (2) only FUV feedback, (3) FUV+EUV feedback, (4) FUV+EUV+SN feedback (the fiducial case), and (5) only SN feedback. Note that the feedback from the initial phase stars continues after $5 \mathrm{Myr}$ in all cases, but the effects of their EUV and SN soon die out, while the impact of the reduced FUV feedback from these stars also gradually declines. ISM properties and star formation activity from 5 to $20 \mathrm{Myr}$ and their dependencies on how feedback is modeled are the focus of this paper.

Figures 1 and 2 show images of the fiducial simulation results, i.e., FUV $+\mathrm{EUV}+\mathrm{SN}$ feedback, after 5, 10, 15, and $20 \mathrm{Myr}$ as viewed from above (top-down view) and from the side (in-plane view) of the disk, respectively. The quantities displayed are the mass surface density structure of the gas, $\Sigma_{g}$, together with locations of young stars, distinguishing those that form after $5 \mathrm{Myr}$ from those that formed earlier; number density of $\mathrm{H}$ nuclei, $n_{\mathrm{H}}$ (this quantity and the following are mass-weighted along the line of sight); gas temperature, $T ; \mathrm{H}_{2}$, 


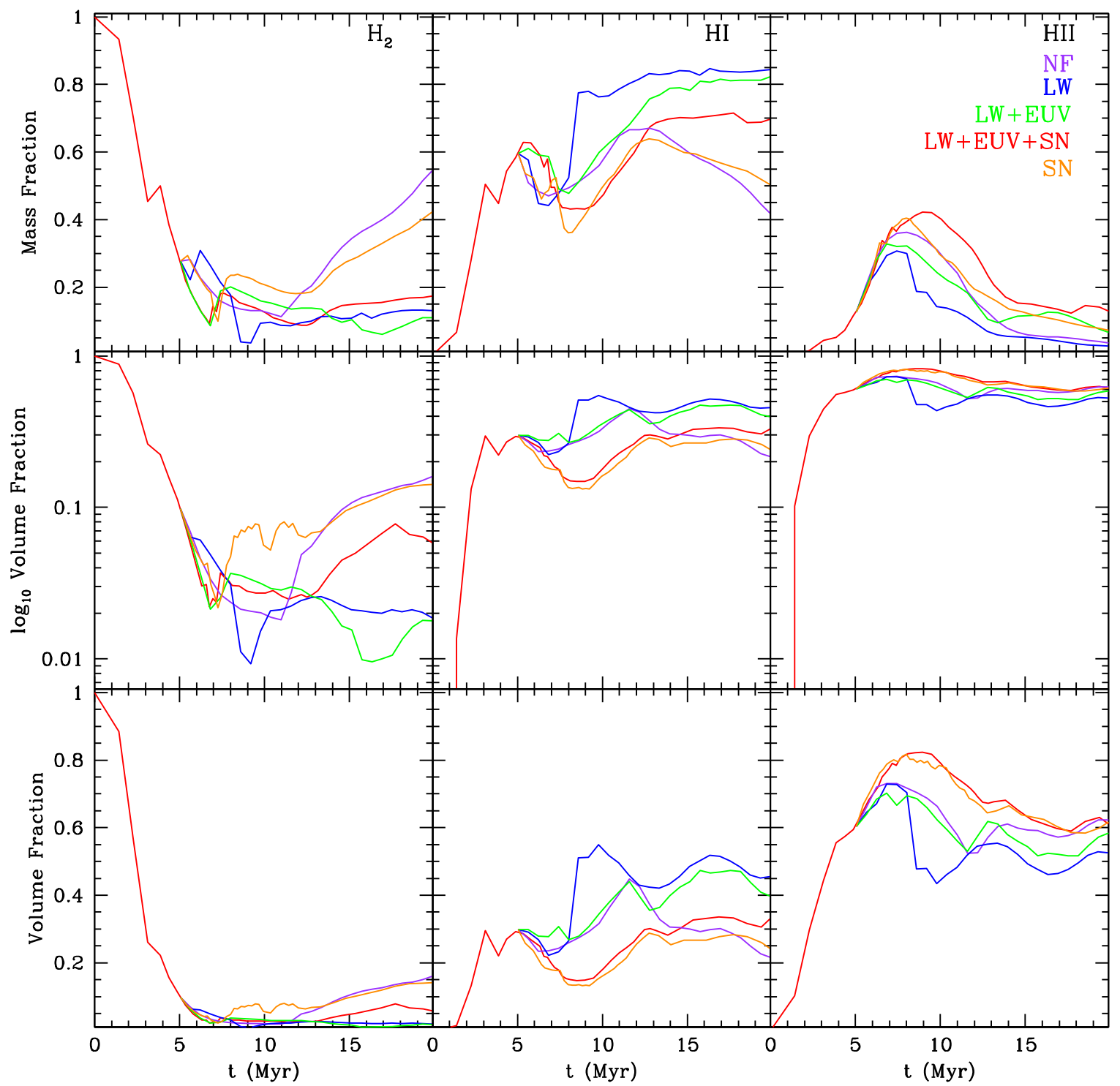

Figure 3. Time evolution of the hydrogen phase mass fractions (top row) and volume fractions (middle and bottom rows) of the 100 pc thick-disk region for all simulations. The fiducial simulation $(\mathrm{LW}+\mathrm{EUV}+\mathrm{SN})$ achieves a quasi-steady state in these phase fractions during the period from $\sim 10$ to $20 \mathrm{Myr}$.

$\mathrm{H}$, and $\mathrm{H}^{+}$mass fractions; $\mathrm{H}_{2}$ dissociating (LW) photon intensities; and H-ionizing (EUV) photon intensities.

Figure 1's top-down view is the clearest way to visualize the evolution of the several initial GMCs that are orbiting in the galactic disk. With the velocity reference frame of the simulation set equal to the orbital velocity of the center of the box, the shear flow causes GMCs on the left, inner-galaxy side to tend to move upwards in the figures, while clouds on the right, outergalaxy side move downwards. However, note that the clouds also inherit random motions, $\sim 10 \mathrm{~km} \mathrm{~s}^{-1}$, from the global galaxy simulation. Periodic boundary conditions are adopted for the sides, while outflow boundary conditions are applied on the top and bottom sides of the cube. Using AMR, finer resolution grids down to $0.5 \mathrm{pc}$ scales are applied (i.e., up to four levels of refinement), which follow fragmentation of the GMCs into dense gas clumps.

Above our adopted threshold density of $n_{\mathrm{H}, *}=10^{5} \mathrm{~cm}^{-3}$ and for gas that is $>90 \%$ molecular, stars are formed with the empirically motivated subgrid model that turns gas into star particles at an efficiency of $2 \%$ per local free-fall time (Krumholz \& Tan 2007), described in Section 2.2. These star particles then act as sources of dissociating, ionizing, and, after a 3 Myr delay, supernova feedback. The radiative feedback can be seen directly in the figures showing the intensities of dissociating and ionizing radiation, which then influence the temperature and chemical state of the gas. The intensity of the UV LW radiation field varies by orders of magnitude, being very high in regions of active star formation. By $20 \mathrm{Myr}$, the median FUV intensity in the $100 \mathrm{pc}$ thick-disk midplane region is about $8.0 G_{0}$.

In Figure 3, we show the time evolution of the mass and volume fractions of the various ISM phases in the $100 \mathrm{pc}$ thick-disk region for the different feedback simulations. For the fiducial simulation at 10,15 , and $20 \mathrm{Myr}$, the ISM has overall mass fractions $X_{\mathrm{H} 2}=0.124,0.154,0.174, X_{\mathrm{H} \mathrm{I}}=0.474,0.695$, 0.699 and $X_{\mathrm{H} \mathrm{II}}=0.402,0.151,0.127$, respectively. The equivalent volume fractions are $V_{\mathrm{H} 2}=0.0273,0.0498,0.0571$, 
(1) NF

(2) LW

(3) LW+EUV (4) LW+EUV+SN

(5) SN

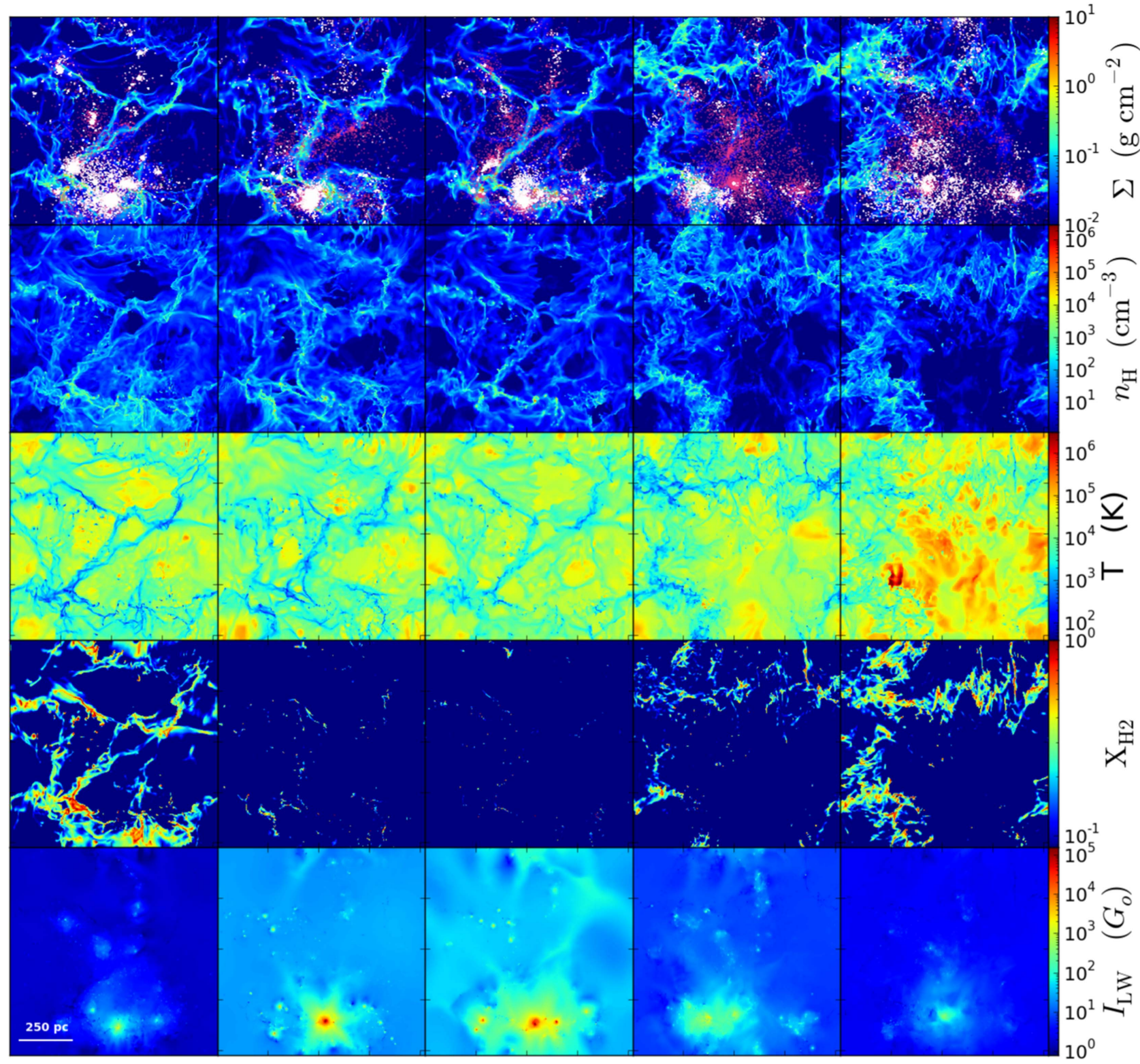

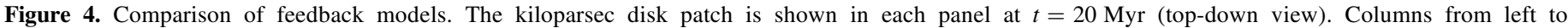

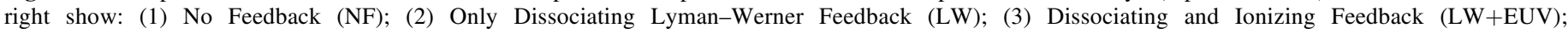

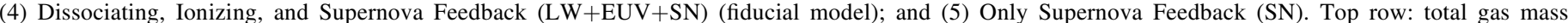

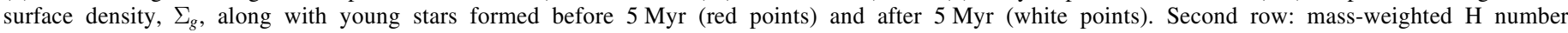

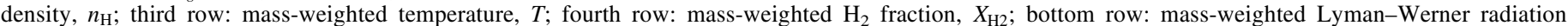
intensity, $I_{\mathrm{LW}}$.

$V_{\mathrm{H}}=0.183,0.326,0.336$, and $V_{\mathrm{H} \text { II }}=0.790,0.624,0.607$ (we note that only a very small fraction of the total gas mass, $\lesssim 3 \%$, is expelled from the simulation box to distances $>500 \mathrm{pc}$ ). After a burst of ionizing feedback, which raises $X_{\mathrm{H} \text { II }}$ to about 0.4 , these mass and volume fractions of the different phases approach relatively constant values as the simulation evolves toward $20 \mathrm{Myr}$, illustrating that by these metrics the ISM reaches a quasi-statistical equilibrium. We note that by $20 \mathrm{Myr}$ the fiducial simulation has a ratio $X_{\mathrm{H} 2} /\left(X_{\mathrm{H} 2}+X_{\mathrm{H} 1}\right) \simeq 0.2$, which is $2-3$ times smaller than the average values inferred inside the solar circle by Koda et al. (2016). We expect that this is due to the moderately elevated values of FUV intensity that are present, i.e., equivalent to $8 G_{0}$, about double that in the inner Galaxy.

Comparing the results of the different feedback simulations in the sequence from Runs 1 to 4 (NF, LW, LW+EUV, $\mathrm{LW}+\mathrm{EUV}+\mathrm{SN}$ ), we see the $\mathrm{H}_{2}$ mass fraction at $20 \mathrm{Myr}$ being reduced to close to the quasi-equilbrium value, mostly by the introduction of LW feedback. On its own, this LW feedback keeps most of the gas in the $\mathrm{H}$ I phase. With the introduction of EUV and SN, a more significant mass fraction of the ionized phase is created. SN-only feedback leads to a much higher $\mathrm{H}_{2}$ mass fraction. 
(1) NF

(2) LW

(3) LW+EUV (4) LW+EUV+SN

(5) SN

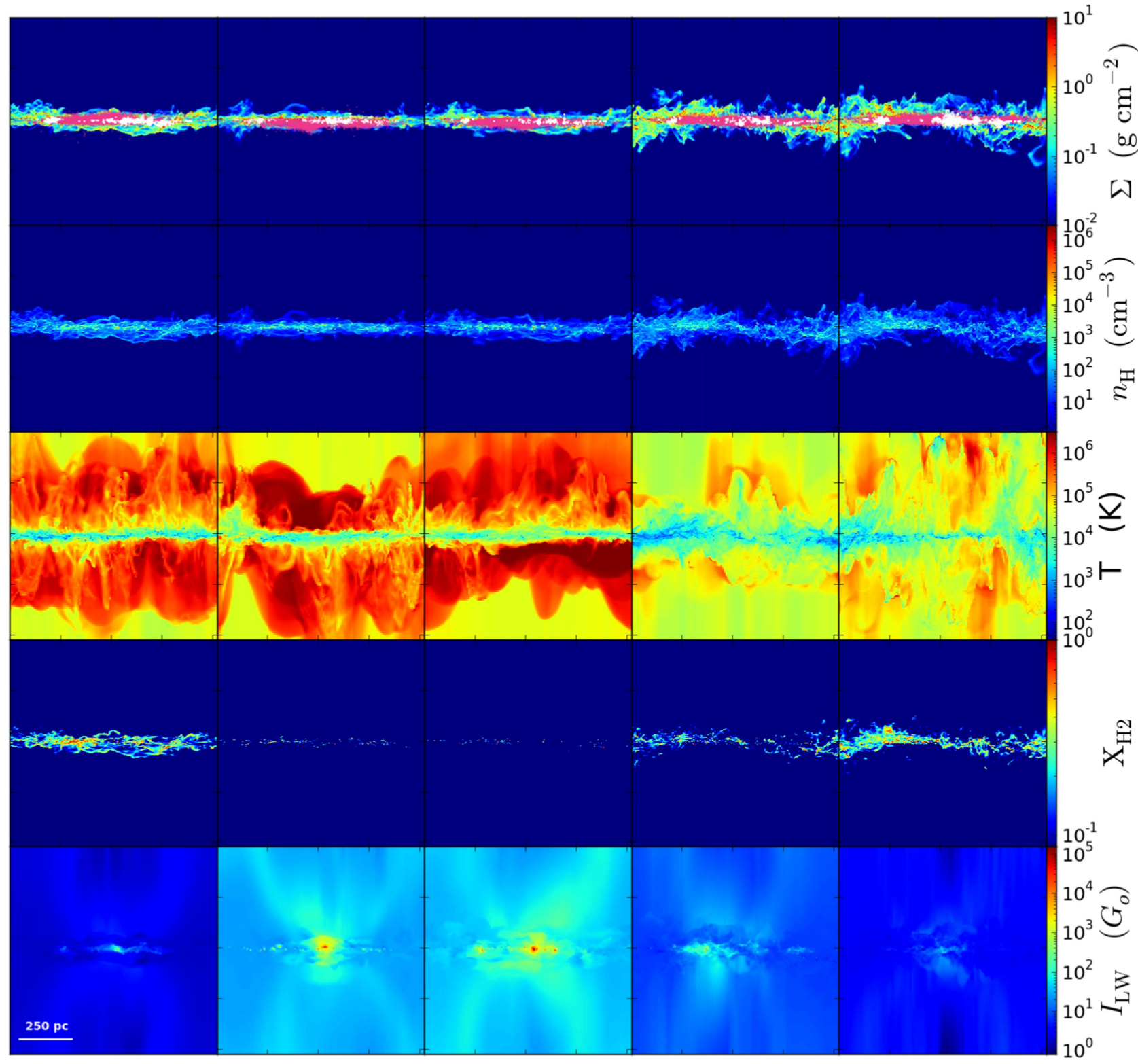

Figure 5. As in Figure 4, but now viewed with an in-plane view, projected along the $y$-axis.

The equivalent results to Figures 1 and 2 for the simulations that have different feedback implementation after $5 \mathrm{Myr}$ are shown in the Appendix, i.e., no feedback (Run NF), only dissociating LW feedback (Run LW), dissociating plus ionizing feedback (Run LW+EUV), and only supernova feedback (Run SN; Figures 11-18). The choice of how feedback is implemented can have a profound effect on the structure of the ISM. This is also illustrated in Figures 4 and 5, which show the results of all of the above simulations side by side after $20 \mathrm{Myr}$ of evolution.

The NF run retains denser clouds and the global ISM is generally cooler, with heat input only from the declining effects of feedback from the initial phase (0-5 Myr formed) stars. Adding LW feedback results in much smaller $\mathrm{H}_{2}$ mass and volume fractions and a generally warmer ISM. However, large-scale distributions of dense gas structures remain relatively unaffected compared to the NF model. Adding EUV feedback continues these trends. Long, $\gtrsim 100$ pc, cold, dense filamentary structures are seen aligned with the galactic plane. Finally, the fiducial model with $\mathrm{LW}+\mathrm{EUV}+\mathrm{SN}$ feedback leads to more disruption in the distribution of the dense gas structures: e.g., filaments tend to be shorter and/or more irregular. There are enhanced $\mathrm{H}_{2}$ fractions in some regions due to the sweeping-up of previously atomic gas. More strongly fluctuating gas potentials also appear to lead to a greater dispersal of the stars that are born in bound star clusters. SN-only feedback leads to much more disruption of the initial molecular clouds, higher fractions of the ISM in the hot $\left(\gtrsim 10^{6} \mathrm{~K}\right)$ phase, but also larger fractions of the molecular phase. The young stars are more dispersed in the SN-only feedback run. 


$$
\begin{aligned}
& \Sigma_{\mathrm{g}}\left(\mathrm{M}_{\odot} \mathrm{pc}^{-2}\right) \\
& \Sigma_{\mathrm{g}}\left(\mathrm{M}_{\odot} \mathrm{pc}^{-2}\right) \\
& \Sigma_{8}\left(\mathrm{M}_{\odot} \mathrm{pc}^{-2}\right) \\
& \Sigma_{8}\left(\mathrm{M}_{\odot} \mathrm{pc}^{-2}\right) \\
& \Sigma_{\mathrm{g}}\left(\mathrm{M}_{\odot} \mathrm{pc}^{-2}\right)
\end{aligned}
$$

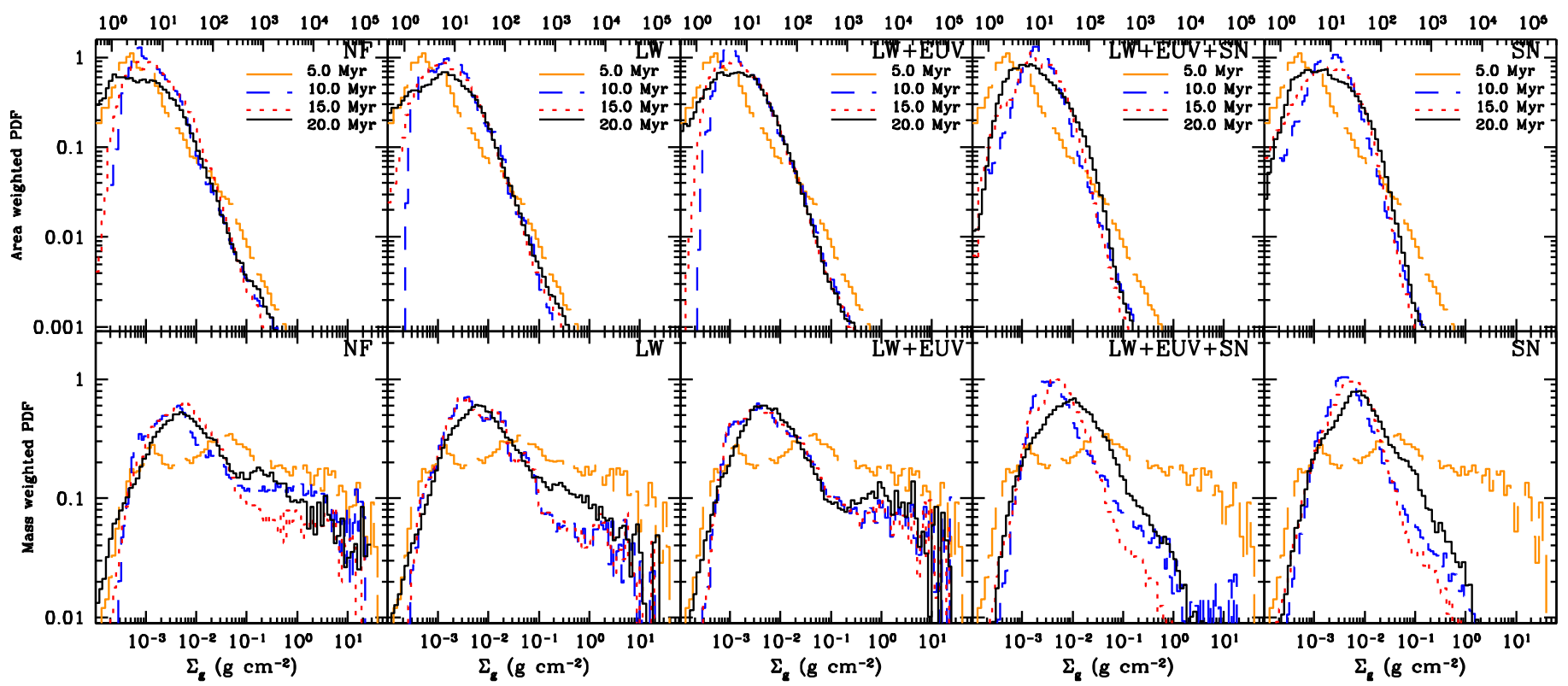

Figure 6. Time evolution from 5 to $20 \mathrm{Myr}$ of the area-weighted (top row) and mass-weighted (bottom row) gas mass surface density probability distribution functions (as viewed from above the disk) for the (from left to right columns) no-feedback run, $\mathrm{LW}$ run, $\mathrm{LW}+\mathrm{EUV}$ run, $\mathrm{LW}+\mathrm{EUV}+\mathrm{SN}$ run, and SN-only run.

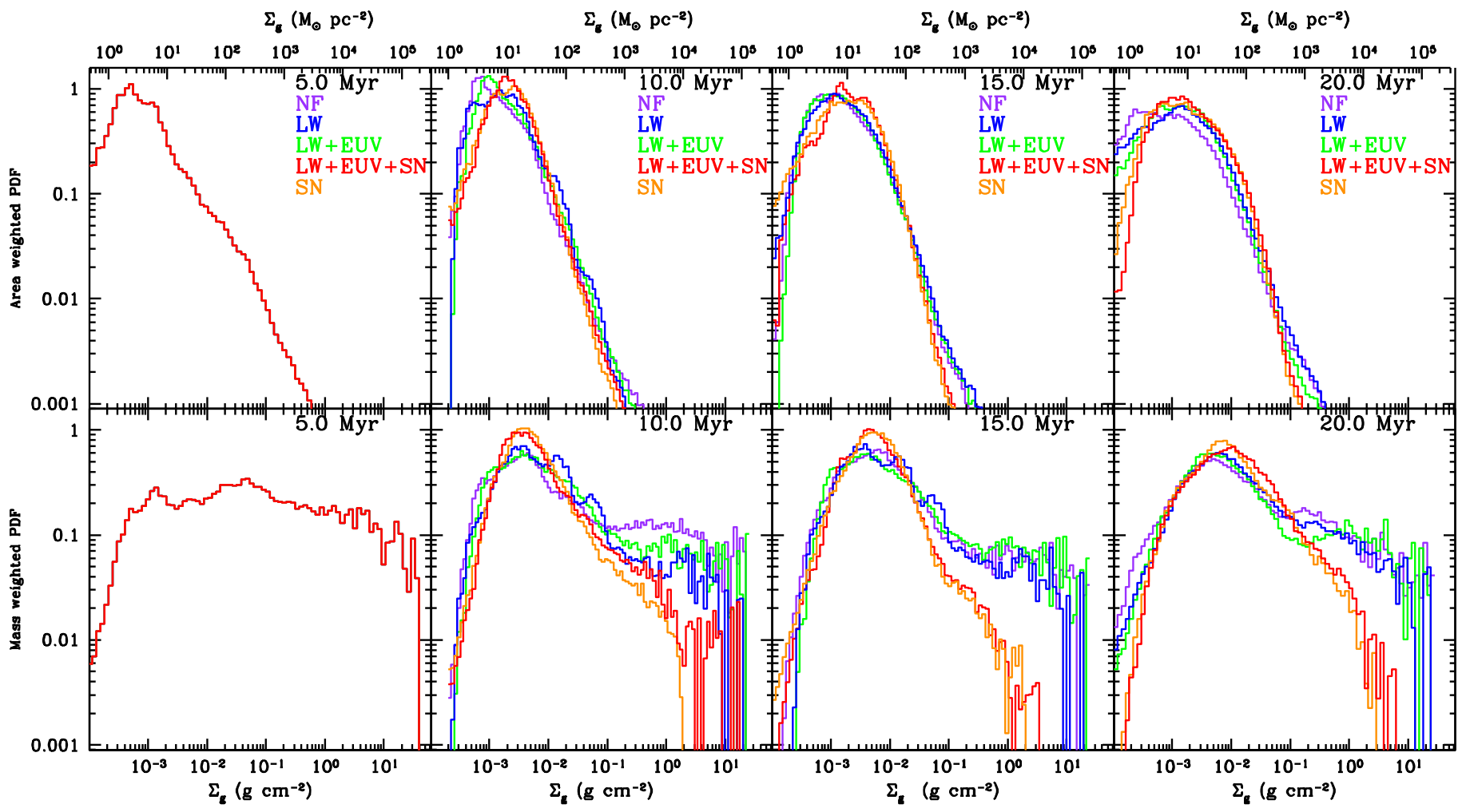

Figure 7. Area-weighted (top) and mass-weighted (bottom) gas mass surface density probability distribution functions (as viewed from above the disk) at 5.0, 10.0, 15.0, and 20.0 Myr for the no-feedback run (purple), LW run (blue), LW+EUV run (green), LW+EUV+SN run (red), and SN-only run (orange).

In Figures 6 and 7, we present comparisons of the area and mass-weighted probability distribution functions (PDFs) of gas mass surface densities, viewed from above the disk, that arise in the different models. These are among the simplest statistical metrics of the ISM, which can be compared to observed systems, especially as we enter the era of full operation of the Atacama Large Millimeter/submillimeter Array that has the ability to resolve $\sim$ parsec scales in nearby galaxies. Figure 6 shows that relatively large changes in the PDFs occur from 5 to $10 \mathrm{Myr}$ in all models and then subsequent evolution is more gradual. The 
(1) NF

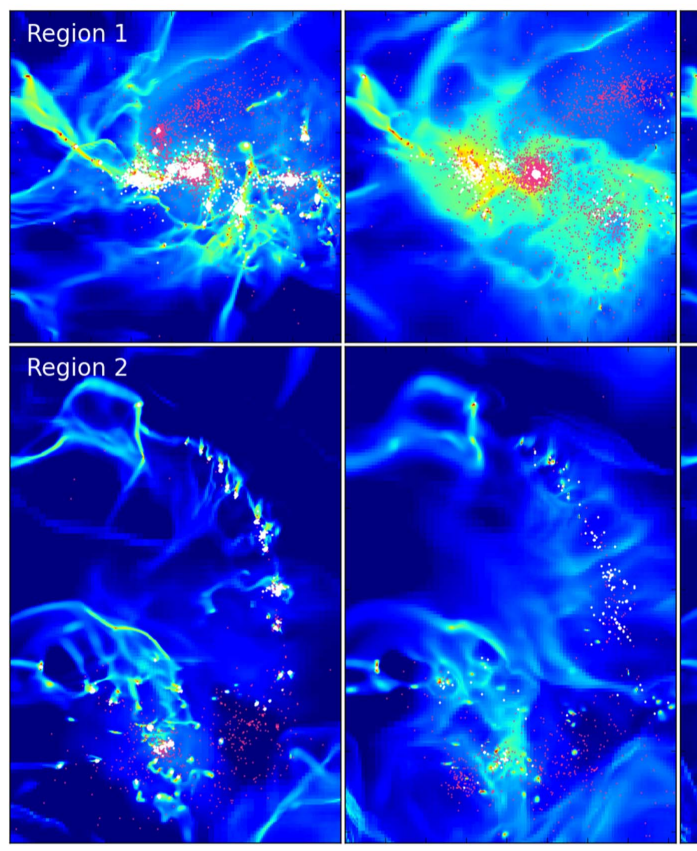

(3) LW+EUV

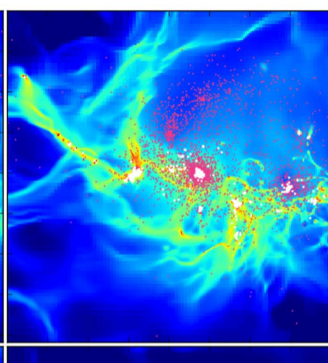

(4) LW+EUV+SN

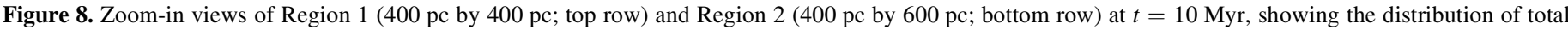

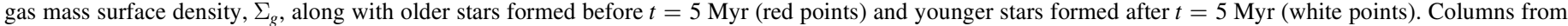

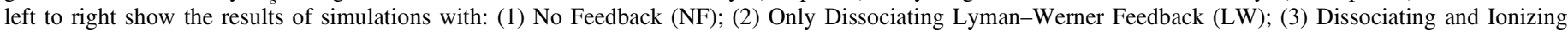

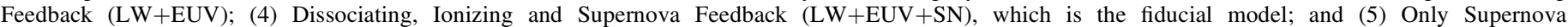
Feedback (SN).

mass fraction at high $\Sigma$ conditions decreases, which is a consequence of both formation of stars and disruption of gas clouds by feedback.

Figure 7 compares the different feedback models at particular times in the simulations, starting from identical initial conditions at $5 \mathrm{Myr}$. The rapid changes from 5 to $10 \mathrm{Myr}$ are again obvious. By $20 \mathrm{Myr}$, the area-weighted $\Sigma$ PDFs in the models with feedback achieve distributions with a single main peak just below $10 M_{\odot} \mathrm{pc}^{-2}$. In the mass-weighted PDFs, the high- $\Sigma$ component is more apparent, and at 15 and $20 \mathrm{Myr}$, we notice quite similar distributions in the NF, LW, and LW + EUV models. However, there can be factors of a few differences in the amount of material with $\Sigma \sim 0.4 \mathrm{~g} \mathrm{~cm}^{-3}$, which is close to the value of individual $0.5 \mathrm{pc}$ cells on reaching the threshold volume density for star formation of $n_{\mathrm{H}, *}=10^{5} \mathrm{~cm}^{-3}$. The effect of SN in the LW+EUV+SN and $\mathrm{SN}$-only runs is to destroy much of the highest $\Sigma$ structures, i.e., $\gtrsim 1 \mathrm{~g} \mathrm{~cm}^{-2}$. Another feature of the PDFs is that those of the $\mathrm{LW}+\mathrm{EUV}+\mathrm{SN}$ and $\mathrm{SN}$-only runs are quite similar to each other.

To better visualize the locations of stars and gas and the effects of different feedback implementations, in Figure 8 we show zoom-ins to two regions at the time of 10 Myr. Region 1, a square patch of $400 \mathrm{pc}$ on a side, is centered in the disk midplane at $(x=+100 \mathrm{pc}, y=-200 \mathrm{pc})$ relative to the center of the kiloparsec box and contains the most vigorous starburst activity. Region 2, which is $400 \mathrm{pc}$ by $600 \mathrm{pc}$ in size and centered at $(x=0 \mathrm{pc}, y=300 \mathrm{pc})$, has a more moderate level of star formation. These regions are the same as those previously analyzed at $10 \mathrm{Myr}$ in the MHD simulations of Van Loo et al. (2015). Recall that each star particle represents a cluster or subcluster with $100 M_{\odot}$ that has formed during the last $10 \mathrm{Myr}$, with the red color for the $0-5 \mathrm{Myr}$ population (common to all runs) and white color for those formed from 5 to $10 \mathrm{Myr}$ and having varying feedback properties. These figures give a dramatic visual illustration of differences in the morphologies of molecular clouds and their star formation activity that result from different implementations of feedback physics. For example, in Region 1, much more massive star clusters form in the no-feedback run, while these are mostly dispersed during the sequence $\mathrm{LW}, \mathrm{LW}+\mathrm{EUV}$, and $\mathrm{LW}+\mathrm{EUV}+\mathrm{SN}$. The $\mathrm{SN}$-only run has a quite different distribution of young stars, which have had the most destructive effects on their natal clouds.

To explore the differences in young stellar populations more quantitatively, we identify the five most massive young star clusters and/or associations in each simulation in Region 1, i.e., a starburst region, selected to each be contained within $\sim 50 \mathrm{pc}^{3}$ scale volumes. For Run NF at $10 \mathrm{Myr}$, we find that the five most massive clusters/associations (only counting stars formed after $5 \mathrm{Myr}$ ) have a mean mass of $1.6 \times 10^{5} \mathrm{M}_{\odot}$. Introducing $\mathrm{LW}$ feedback reduces this to $5.7 \times 10^{4} M_{\odot}$; LW+EUV feedback further reduces this to $5.1 \times 10^{4} M_{\odot}$; and $\mathrm{LW}+\mathrm{EUV}+\mathrm{SN}$ feedback reduces this to $1.2 \times 10^{4} M_{\odot}$. On the other hand, the $\mathrm{SN}$-only feedback simulation produces the five most massive clusters with mean mass of $7.3 \times 10^{4} M_{\odot}$. Thus, the way in which feedback is implemented can have a dramatic impact on the clustering of star formation.

\subsection{The Effect of Feedback on SFRs}

We now examine the SFRs that occur during the simulations and the effects of different feedback models. The time evolution of the SFR in the whole kiloparsec patch is shown 


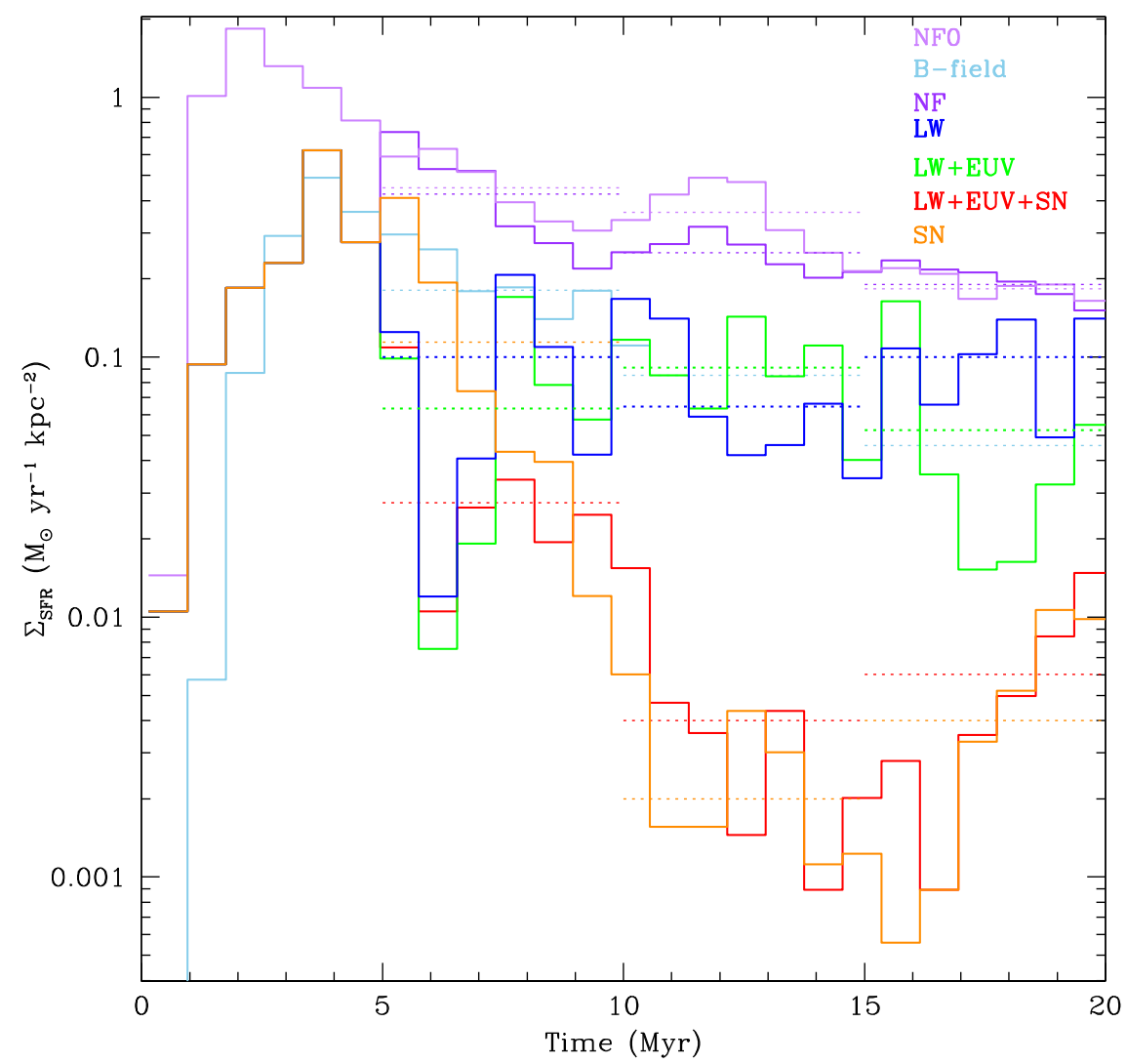

Figure 9. Time evolution of star formation rate per unit area, $\Sigma_{\mathrm{SFR}}$, of the whole kiloparsec patch of the disk for Run NF0 (light purple), Run NF (purple), Run LW (blue), Run LW+EUV (green), Run LW+EUV+SN (red), and Run SN (orange). The averages from 5-10 Myr, 10-15 Myr, and 15-20 Myr are indicated with horizontal dotted lines. The no-feedback run (Van Loo et al. 2015) with magnetic fields, which was carried out to 10 Myr, is also shown (light blue), along with estimates of its average SFRs from 10 to $20 \mathrm{Myr}$.

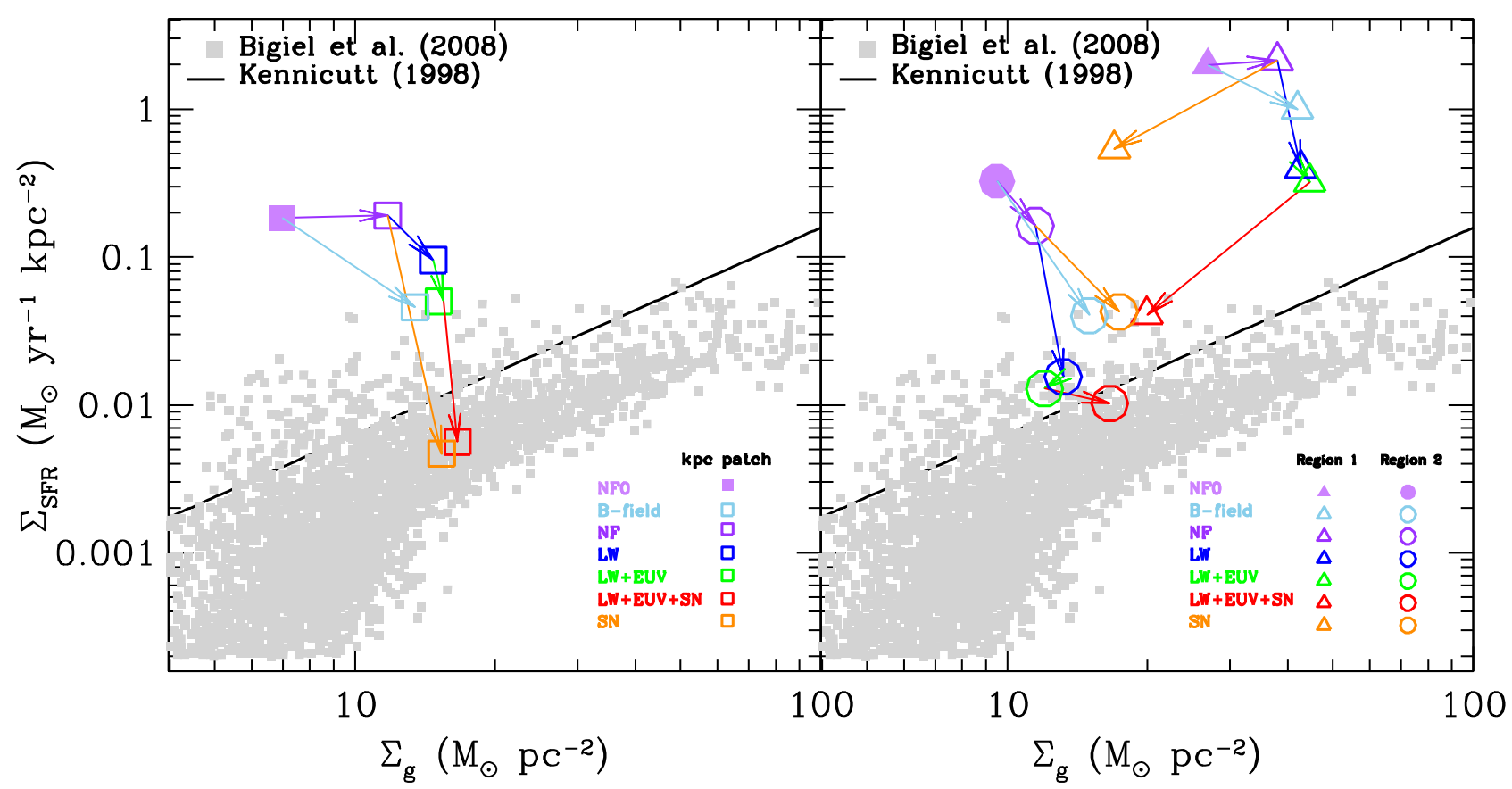

Figure 10. Star formation rate per unit area vs. total gas mass surface density. Left panel shows results for the whole kpc patch of the disk with $\Sigma_{g}$ evaluated at $20 \mathrm{Myr}$ and $\Sigma_{\text {SFR }}$ being the average from 15 to 20 Myr. The right panel shows results for Regions 1 and 2 with $\Sigma_{g}$ evaluated at 10 Myr and $\Sigma_{\text {SFR }}$ being the average from 5 to 10 Myr. The different simulation runs are labeled in the legends (see text for description of the model sequences). Observational data from Bigiel et al. (2008) are plotted as gray squares (annuli of disk galaxies), while the empirical power-law fit to the galactic and circumnuclear disk averages of Kennicutt (1998) is shown with a black solid line. 
in Figure 9, averaging over time intervals of just under 1 Myr. Here we also show the results of a run, NF0, which has no feedback at all for the entire period from 0 to $20 \mathrm{Myr}$. This provides a baseline result against which the other simulations can be compared. In addition, we plot the 0-10 Myr SFR results of the MHD simulation of Van Loo et al. (2015), in which the galactic disk patch is initially threaded with a uniform $B$ field of strength of $10 \mu G$, along with an extrapolated SFR, assuming exponential decay, from 10 to 20 Myr. As discussed by Van Loo et al. (2015), magnetic fields help support the clouds against collapse and lead to reductions in SFRs of factors of a few.

For the feedback simulation runs presented in this paper, all implement the "initial feedback model" from 0 to $5 \mathrm{Myr}$, designed to reach a realistic FUV intensity by this time. During this phase, we see the suppression of the SFR by about a factor of 10 in the first few Myr, then by smaller factors. After $5 \mathrm{Myr}$, the no-feedback case eventually converges toward an SFR approximately equal to the NF0 run as the effects of stars formed in the initial feedback phase die out. Introducing only LW feedback leads to a reduction of factors of several in SFR compared to Run NF over most of the $20 \mathrm{Myr}$ evolution. However, the SFR now also shows much larger fluctuations, which indicates the bursty nature of star formation and subsequent feedback that is occurring in the simulated disk patch. We will thus typically consider SFRs from the simulations averaged over 5 Myr periods. The SFRs in the three $5 \mathrm{Myr}$ period averages from 5 to $20 \mathrm{Myr}$ are fairly constant at a level of about $\Sigma_{\mathrm{SFR}}=0.1 M_{\odot} \mathrm{yr}^{-1} \mathrm{kpc}^{-2}$.

Continuing along the sequence of feedback models, the introduction of ionizing feedback in Run LW+EUV leads to SFRs that, on average, are only modestly smaller than Run LW, and sometimes can even be higher, e.g., from 10 to $15 \mathrm{Myr}$. The final step to reaching the fiducial model is the introduction of supernova feedback, i.e., Run $\mathrm{LW}+\mathrm{EUV}+\mathrm{SN}$, which leads to a significant further reduction in the SFRs. The global evolution of the SFR appears to show a longer period systematic fluctuation, decreasing from an initial relatively high value at $5 \mathrm{Myr}$ by almost a factor of 100 by the time around $15 \mathrm{Myr}$, before increasing again to a value around $\Sigma_{\mathrm{SFR}}=0.01 M_{\odot} \mathrm{yr}^{-1} \mathrm{kpc}^{-2}$ by 20 Myr. However, the two $5 \mathrm{Myr}$ period averages from 10 to $20 \mathrm{Myr}$ are relatively similar at about half this value. Note that the absolute amount of reduction of $\Sigma_{\mathrm{SFR}}$ due to the introduction of $\mathrm{SN}$ feedback in this sequence is about the same as that caused by the earlier introduction of LW feedback, relative to the NF case.

Finally, the SN-only feedback run shows an initially relatively high SFR just after $5 \mathrm{Myr}$, which is understandable given the 3 Myr delay associated with this feedback. However, the SFR then declines over the next $10 \mathrm{Myr}$ by about two orders of magnitude, before rising more modestly again after 15 Myr. This SFR history is very similar to that of the fiducial run with full feedback $(\mathrm{LW}+\mathrm{EUV}+\mathrm{SN})$, which is consistent with the very similar PDFs of gas mass surface density seen in these models at late times (Figure 7).

Figure 10(a) shows the results of these simulations on a "Kennicutt-Schmidt" (KS) diagram of $\Sigma_{\text {SFR }}$ versus $\Sigma_{g}$, i.e., the total gas mass surface density. The left panel shows conditions averaged over the whole kiloparsec patch of the simulations, with $\Sigma_{\mathrm{SFR}}$ evaluated as the average from 15 to
$20 \mathrm{Myr}$, i.e., when the statistical properties of the ISM appear to have mostly stabilized (Section 3.1). The value of $\Sigma_{g}$ is that observed at the end of this period, i.e., at $20 \mathrm{Myr}$. This method of constructing the KS diagram is similar to that adopted by observational studies, which measure current gas properties but which evaluate SFRs from the presence of massive star populations that are sensitive to the SFR averaged over the last several Myr. The observational measurements of Kennicutt (1998) and Bigiel et al. (2008), the latter averaging over scales of about $1 \mathrm{kpc}$, are plotted in Figure 10.

We have connected the results of the simulations with a sequence of arrows that shows the effect of adding particular physics. The pure no-feedback result, NF0, is in the upper left with a high SFR and, by $20 \mathrm{Myr}$, a much reduced gas content. The effect of magnetizing this disk with a $10 \mu G$ field strength is shown by the light blue arrow connecting to the extrapolated results of Van Loo et al. (2015). The purple arrow to Run NF illustrates the effect of the initial feedback model implemented from 0 to $5 \mathrm{Myr}$, but then with feedback turned off. The SFR near the end of the simulation is very similar to that of NF0, even though the disk has retained more gas. Adding LW feedback brings the SFR down by just over a factor of two and further raises the gas mass surface density. Ionization has a more modest effect on SFR, with the remaining gas content (set mostly by earlier SFRs) hardly affected. At the end of the sequence, the fiducial model of $\mathrm{LW}+\mathrm{EUV}+\mathrm{SN}$ feedback brings the SFR down by about a factor of 10 at a slightly larger gas content. We see that the fiducial model sits in the middle of the distribution of observed SFRs of Bigiel et al. (2008). The effect of the SNonly model is shown by the orange arrow from NF. Overall, it causes a similar reduction in SFR and increase in retained gas content as the sequence to the fiducial model.

Figure 10(b) shows similar results, but now for Region 1 (starburst conditions) and Region 2 (normal disk conditions; see Figure 8 ). Note that these regions were originally defined at 10 Myr in the simulations of Van Loo et al. (2013), and were also analyzed at this time in the MHD simulations of Van Loo et al. (2015). By $20 \mathrm{Myr}$, the nature of these regions, such as their gas content, undergoes dramatic evolution. Thus, we also analyze the properties of these regions at $10 \mathrm{Myr}$, i.e., the time at which $\Sigma_{g}$ is evaluated, with the SFR being the average from 5 to 10 Myr. We note that this means conditions in the ISM in these regions have not reached quasi-statistical equilibrium. However, on these scales of only several hundred parsecs, such equilibrium is in any case not likely to be achieved when averaging over $5 \mathrm{Myr}$ timescales. Observationally, at these scales of several hundred-parsec-sided regions, a greater dispersion in the normalization of the $\mathrm{KS}$ relation has been reported (Leroy et al. 2013).

Region 2, which is $600 \mathrm{pc}$ by $400 \mathrm{pc}$ in extent and with average conditions quite similar to those of the kiloparsec patch as a whole, shows qualitatively similar behavior. However, LW feedback is seen to play a relatively more important role here along the sequence to the fiducial model of $\mathrm{LW}+\mathrm{EUV}+\mathrm{SN}$. The fiducial model rests at a location slightly elevated in its normalization compared to the median of the data, although still within the range of the observed scatter in the KS relation. Since Region 2 is centered on a GMC complex, this slight enhancement is also consistent with theoretical expectations, 
given the clustered nature of star formation in disk galaxies. In Region 2, the $B$-field model also leads to a significant reduction in SFR, although not as much as that due to the fiducial feedback model. The SN-only feedback model is also not as effective as the full radiative feedback plus $\mathrm{SN}$ model in this case.

Region 1, centered on a very dense grouping of GMCs that are also interacting, shows very high SFR surface densities. The NF0 and NF models have $\Sigma_{\mathrm{SFR}} \simeq 2 M_{\odot} \mathrm{yr}^{-1} \mathrm{kpc}^{-2}$, with the latter retaining a gas mass surface density of about $40 M_{\odot} \mathrm{pc}^{-2}$ at $10 \mathrm{Myr}$. LW feedback brings the SFR down by about a factor of five, with the addition of ionization causing only a minor reduction beyond this. The final $\mathrm{LW}+\mathrm{EUV}+\mathrm{SN}$ model has its SFR reduced further by a factor of 10 to $\Sigma_{\mathrm{SFR}} \simeq 0.04 M_{\odot} \mathrm{yr}^{-1} \mathrm{kpc}^{-2}$. However, note the largest reduction of SFR in absolute terms resulted at the stage of introduction of FUV $\mathrm{H}_{2}$ dissociating feedback. The introduction of SN leads to a dramatic reduction in $\Sigma_{g}$, i.e., by about a factor of three, which is caused by the expulsion of gas from Region 1 to its immediate surroundings. This illustrates the inherent difficulties of measuring the KS relation on these small scales of several hundred parsecs. Still, the final location of Region 1 in the KS diagram for the fiducial model is at a normalization that is consistent with the upper envelope of the observational data, as might be expected for a starburst region.

Finally, we note that the introduction of a magnetic-field support affects the SFR of Region 1 only by about a factor of two, a smaller factor than in Region 1 and in the kiloparsec patch as a whole. This is because the dense GMCs in Region 1 would need stronger $B$-field strengths to resist collapse to the same level as the lower density GMCs in the other parts of the disk. SN-only feedback reduces SFRs by factors of a few and, like the case of $\mathrm{LW}+\mathrm{EUV}+\mathrm{SN}$, expels most of the gas from the region. The final location of the SN-only model in the KS diagram is at a level that is about 10 times higher than the upper envelope of the observed data.

\section{Discussion and Conclusions}

We have found that the combined effects of dissociating, ionizing, and supernova feedback have a dramatic influence on the structure and star formation activity of the interstellar medium of a disk galaxy. The relative mass and volume fractions of different phases of the ISM approach quasi-static values after $20 \mathrm{Myr}$ of evolution of the simulated kiloparsec patch of the disk. The detailed structure of the ISM, especially dense, molecular clouds, and the spatial distribution of young stellar populations varies significantly with how feedback is implemented, as we have also seen in comparison to a supernova-only feedback model.

Of the forms of feedback we have investigated in the sequence of building our fiducial model, it is the introduction of FUV radiation that dissociates $\mathrm{H}_{2}$ molecules and heats the gas, which typically plays the largest role in reducing absolute SFRs from those seen in simulations with no feedback from the newly forming stars. These reduction factors are similar to those seen in models including magnetic support from an ISM magnetized at a level of $10 \mu \mathrm{G}$. However, the full combination of radiative and mechanical feedback from supernovae of our fiducial model is needed to bring these overall SFRs down to values similar to those observed in real disk systems. Averaged on kiloparsec scales, the SFRs resulting from the SN-only feedback models are very similar to those achieved in our fiducial simulation, even though these involve large differences in the chemical and temperature structures of the gas and in the spatial distribution of young stars. Also, in smaller scale regions centered on GMC complexes, there are significant differences in the SFRs between the SN-only case and the full feedback model. These results illustrate the importance of considering FUV feedback in understanding the star formation activity of galactic disk systems. Similar conclusions have been reached by Peters et al. (2017) in their lower (4 pc) resolution studies of a smaller $(0.5 \mathrm{kpc}$ by $0.5 \mathrm{kpc})$ disk patch, which builds on the previous study of a stellar wind and supernova feedback model by Gatto et al. (2017).

The simulations presented in this paper of course have their limitations and there is scope for improvement, which may influence the final results. For example, it will be necessary to combine these feedback models with magnetized ISM simulations. Additional feedback effects such as protostellar outflows from forming stars, stellar winds, and radiation pressure will also need to be considered. The momentum input from these sources can be comparable to that from supernovae, although in the case of outflows and winds, much of this momentum may be lost in oppositely directed flows from a distributed population of stars. Also, initial test simulations including radiation pressure by J. Rosdahl et al. (2017, in preparation) indicate that it plays a relatively minor role on the scale of this kiloparsec patch. On smaller scales, radiation pressure has been studied by, e.g., Krumholz \& Matzner (2009), who find it can be important feedback in modifying $\mathrm{H}$ II region dynamics around massive star clusters. The contribution of relativistic cosmic rays, which are known to have a similar energy density to magnetic and thermal pressures in the local Galactic ISM, will also need to be included. To constrain these more complex models and their particular parameter choices against observed systems, we will also need to examine more detailed statistical metrics, e.g., the structural, kinematic, and chemical properties of the ISM, in addition to SFR activity. Improved resolution down to the core scale of individual star formation is also highly desirable, especially for the resolution of the early stages of ionizing feedback, which will then also necessitate a fully stochastic treatment of the initial stellar mass function of massive stars. On the other hand, the region we have simulated is relatively limited in spatial extent, which may lead to stochastic effects related to the particular GMCs that are included in the volume. Future work should also aim to expand the number of different realizations simulated, the size of the simulated volume, and also explore the effects of a range of galactic environments.

\section{Appendix Additional Simulation Results}

The equivalent results to Figures 1 and 2 for the simulations that have different feedback implementation after 5 Myr are shown in Figures 11-18. 


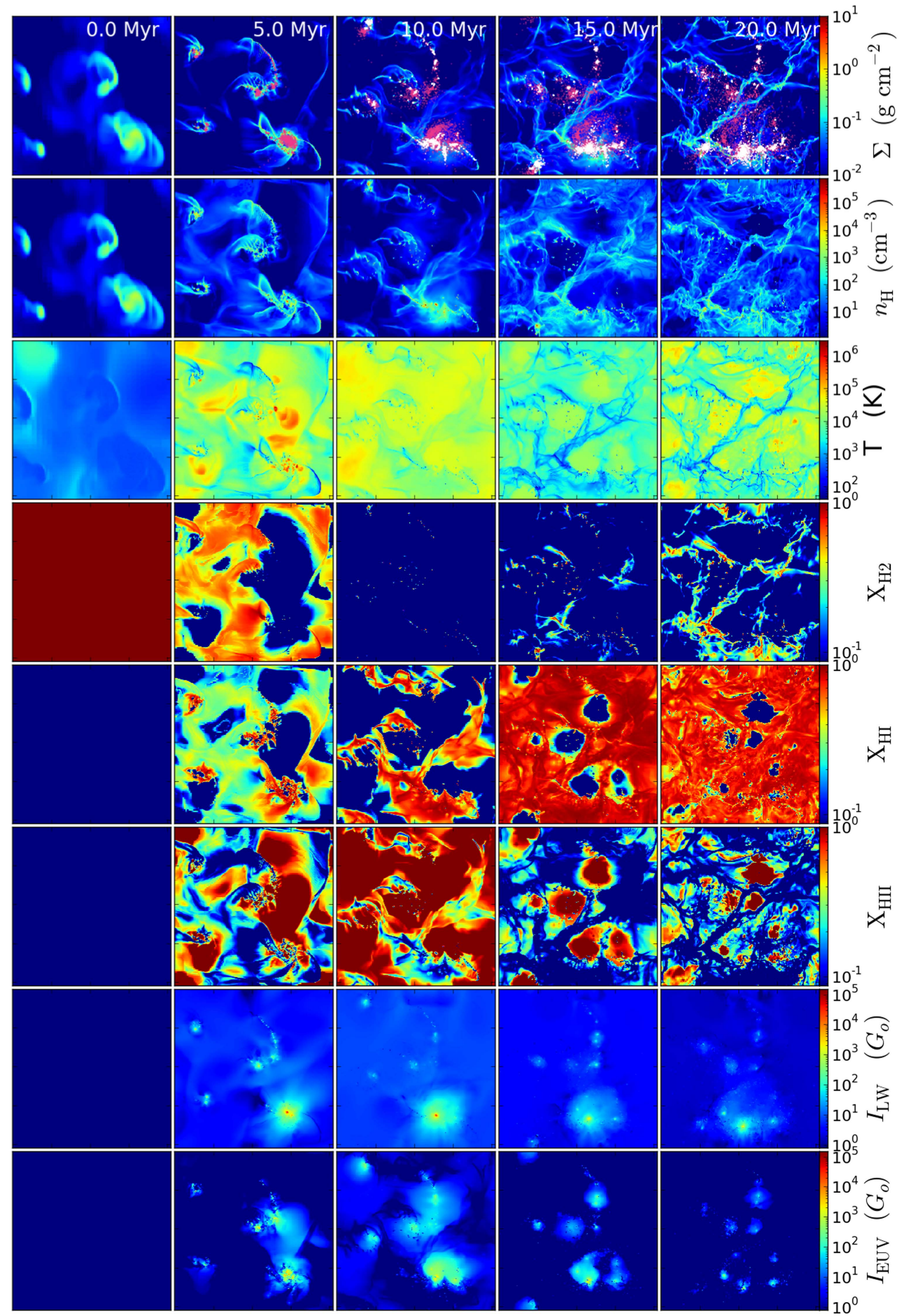

Figure 11. Same as Figure 1, but for the No-feedback simulation (Run NF). 


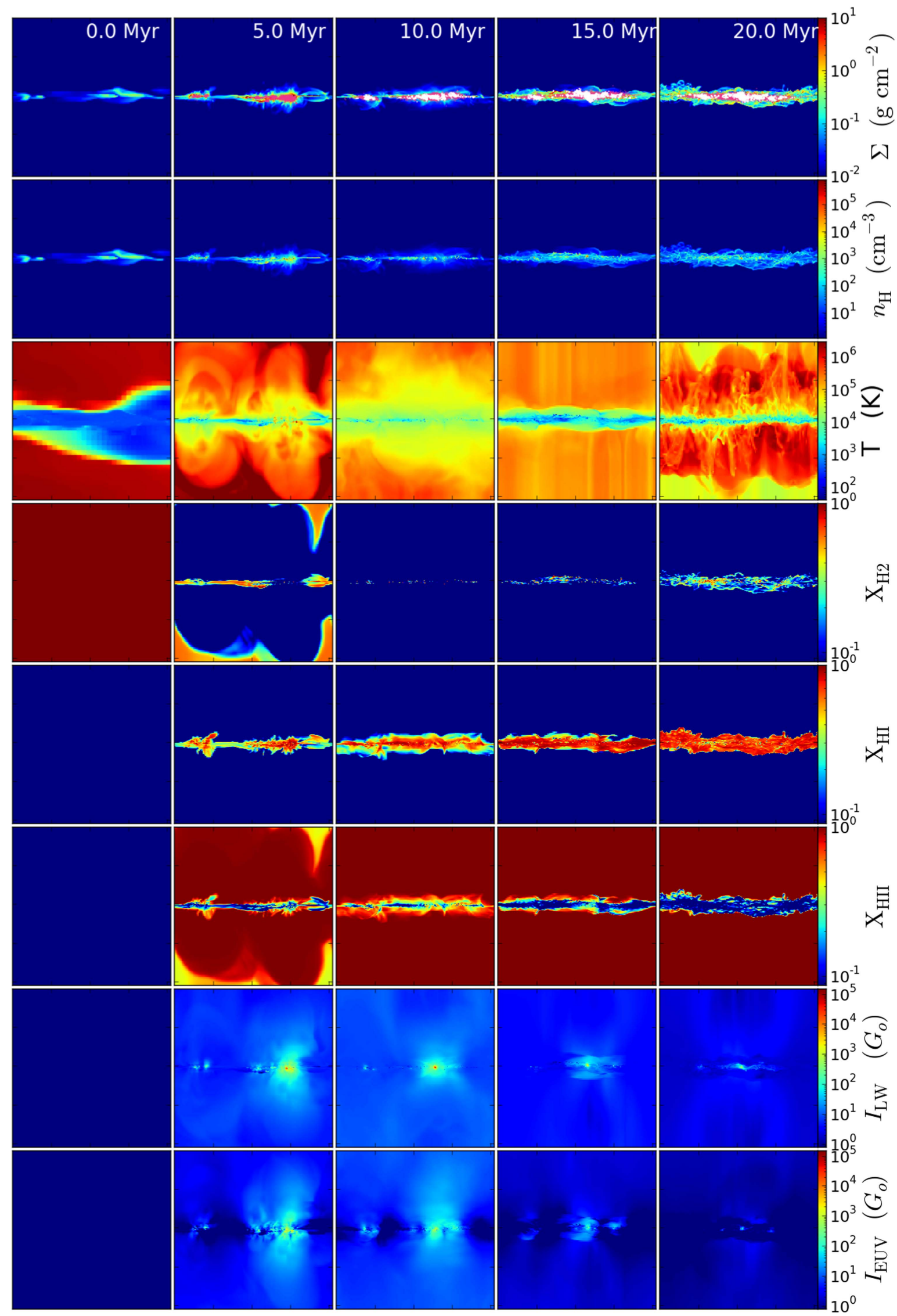

Figure 12. Same as Figure 1, but for the No-feedback simulation (Run NF). 


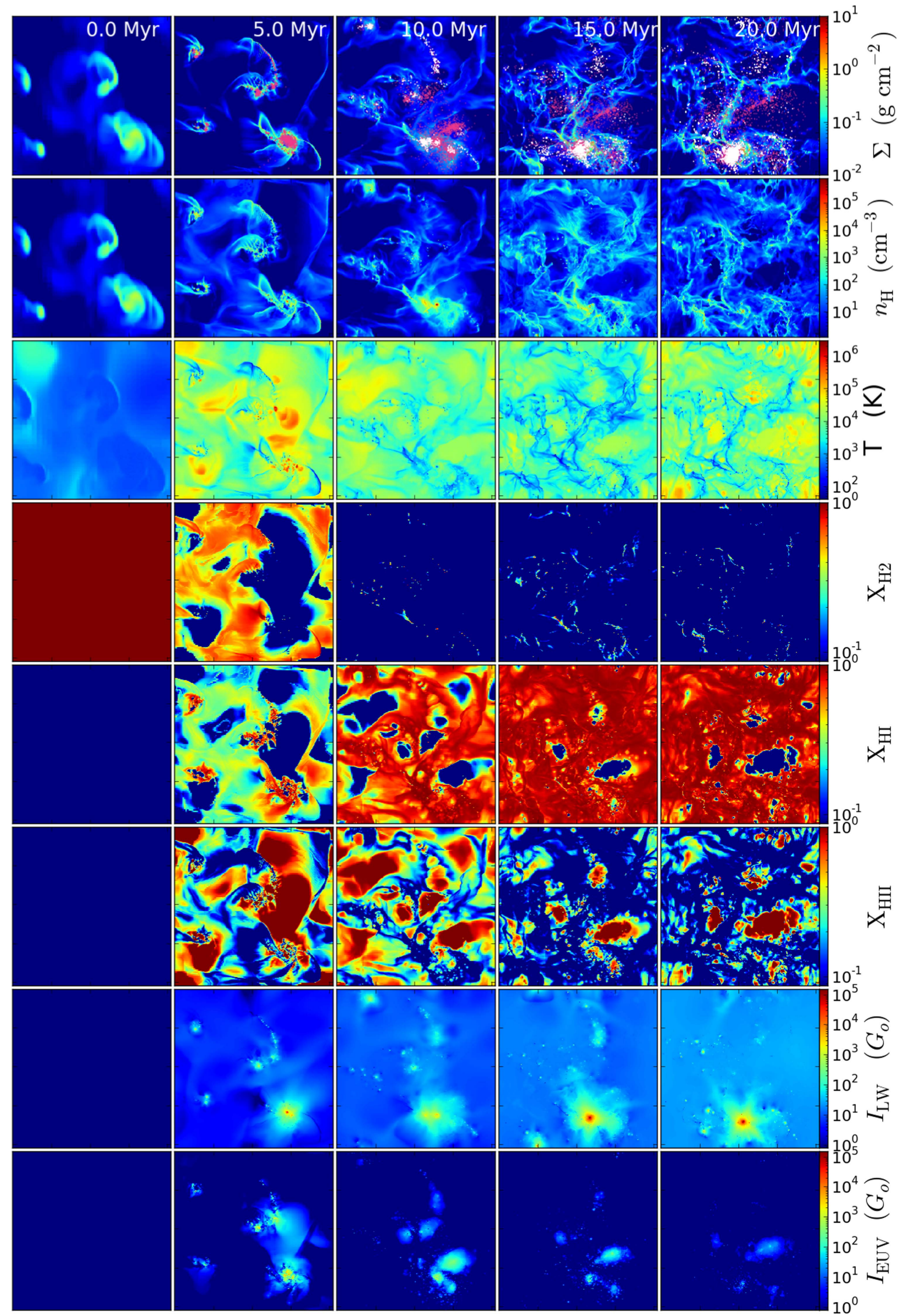

Figure 13. Same as Figure 1, but for the Lyman-Werner only simulation (Run LW). 


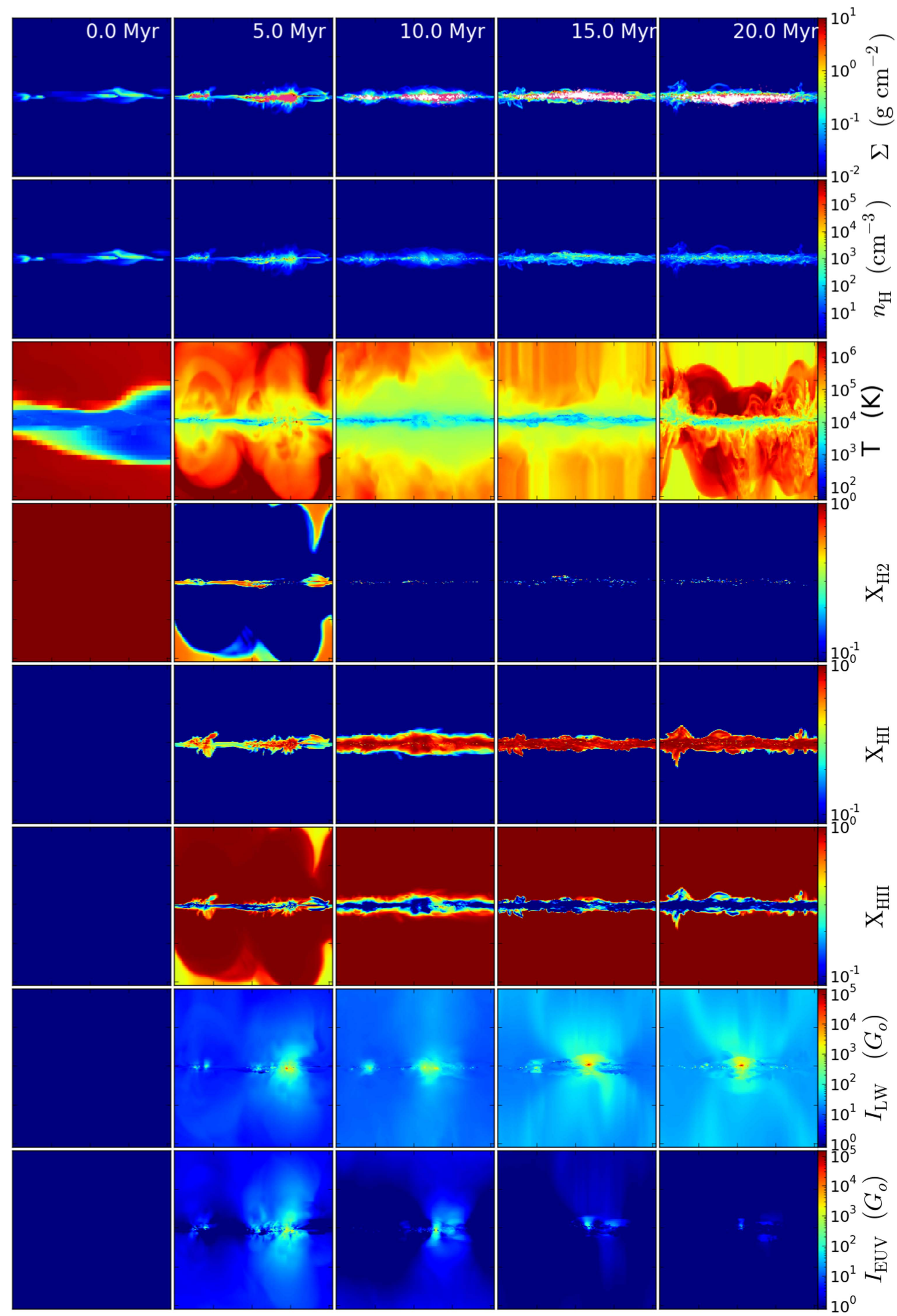

Figure 14. Same as Figure 1, but for the Lyman-Werner only simulation (Run LW). 


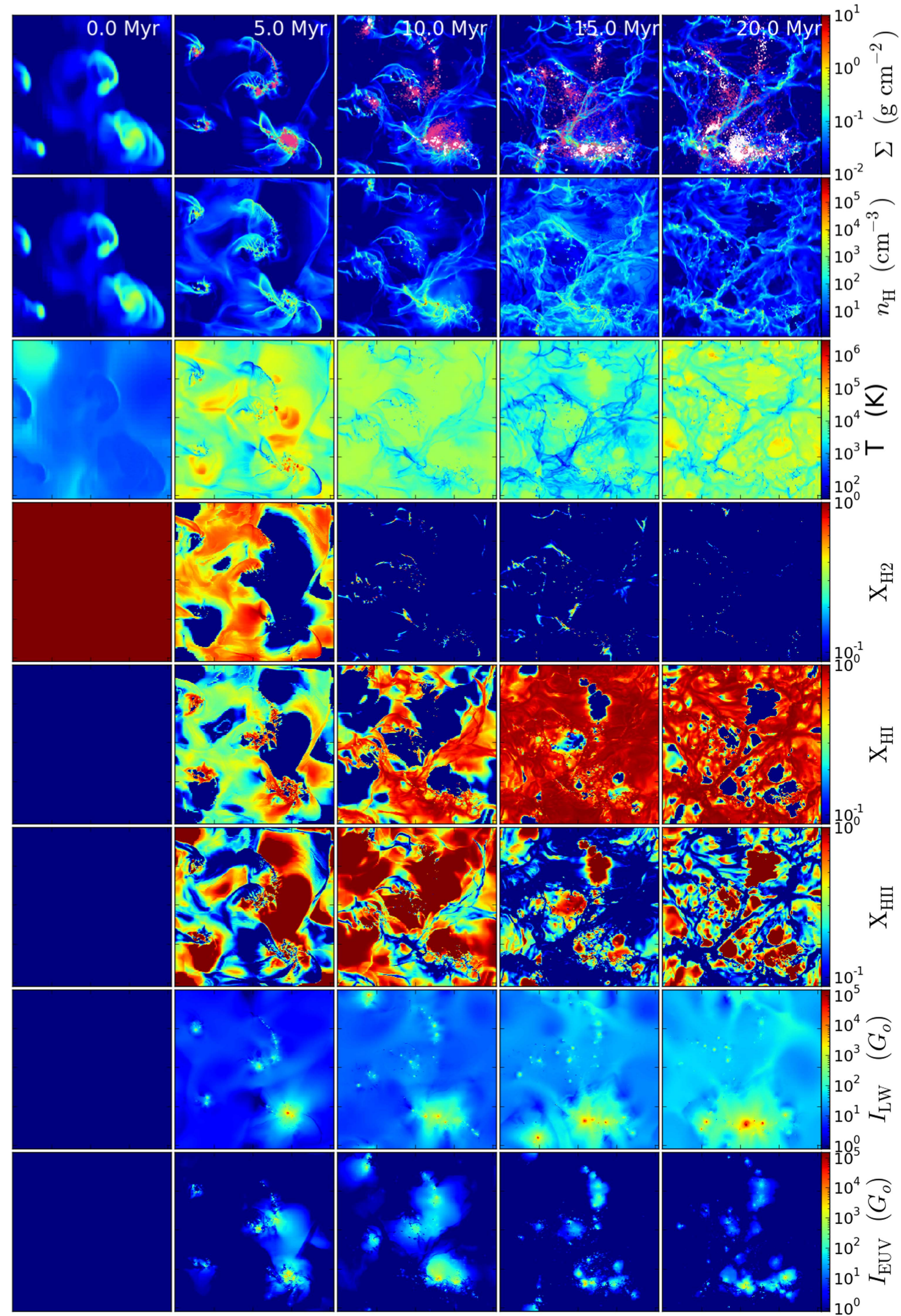

Figure 15. Same as Figure 1, but for the Lyman-Werner and Ionization simulation (Run LW+EUV). 


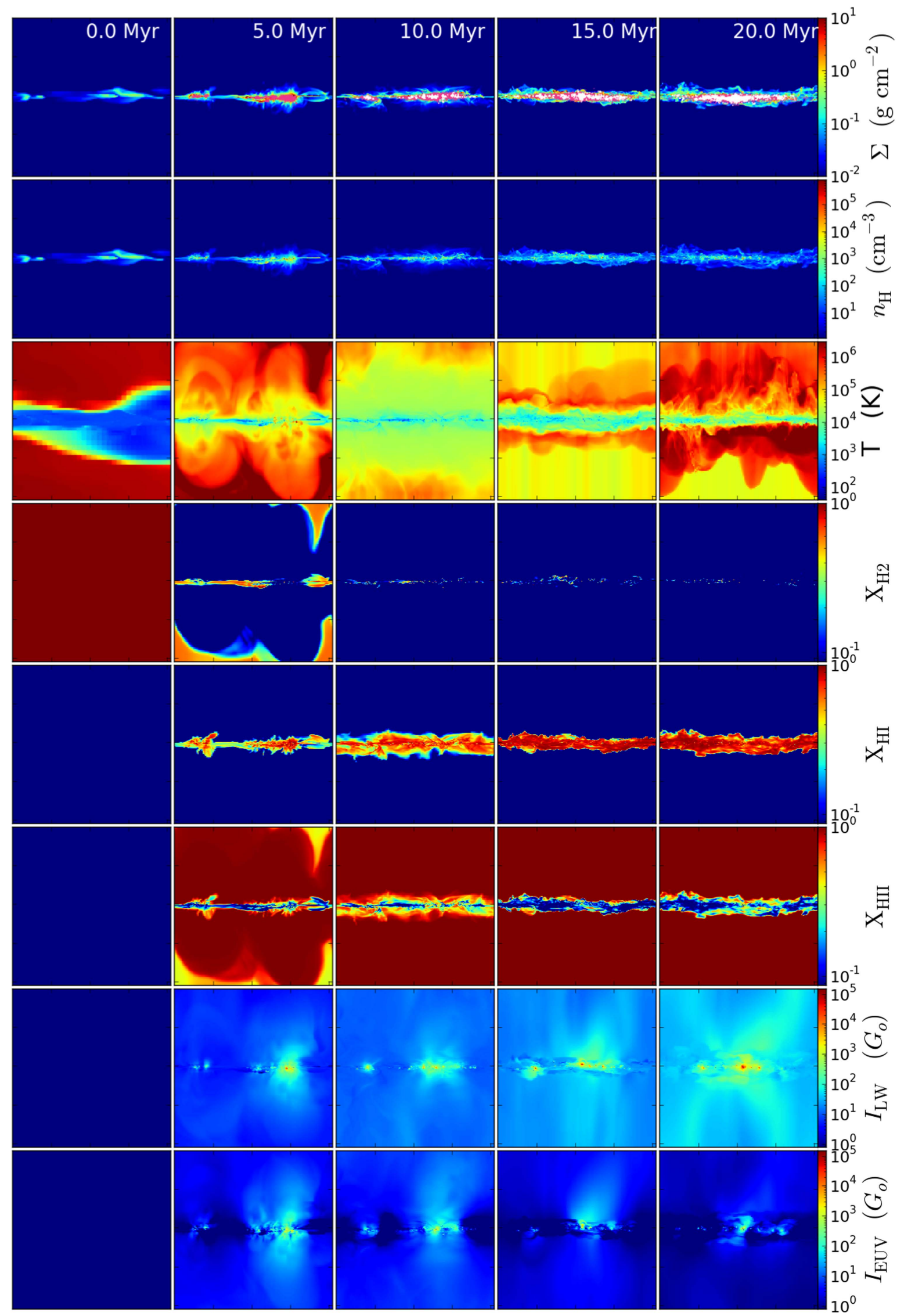

Figure 16. Same as Figure 1, but for the Lyman-Werner and Ionization simulation (Run LW+EUV). 


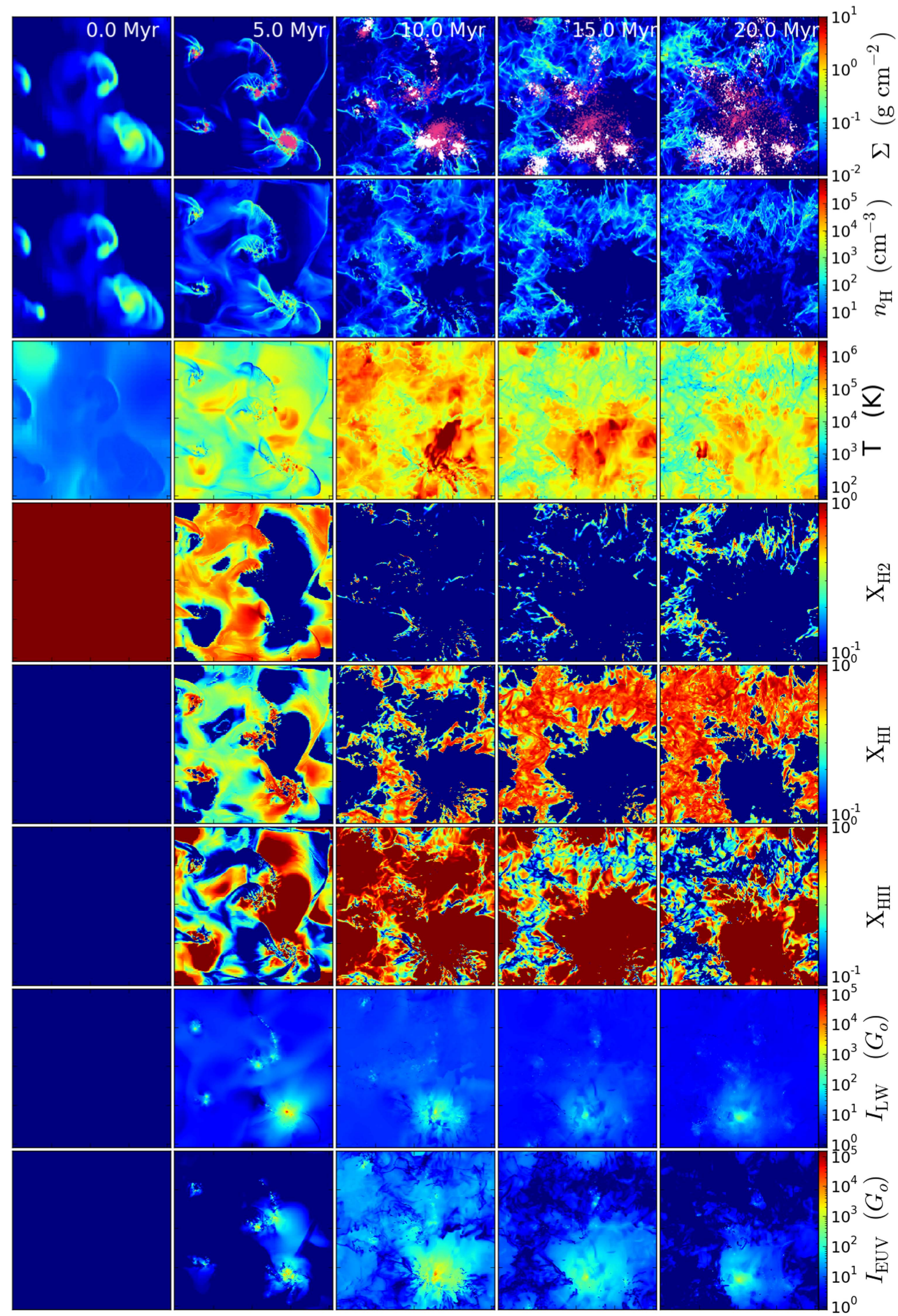

Figure 17. Same as Figure 1, but for the Supernova-only simulation (Run SN). 


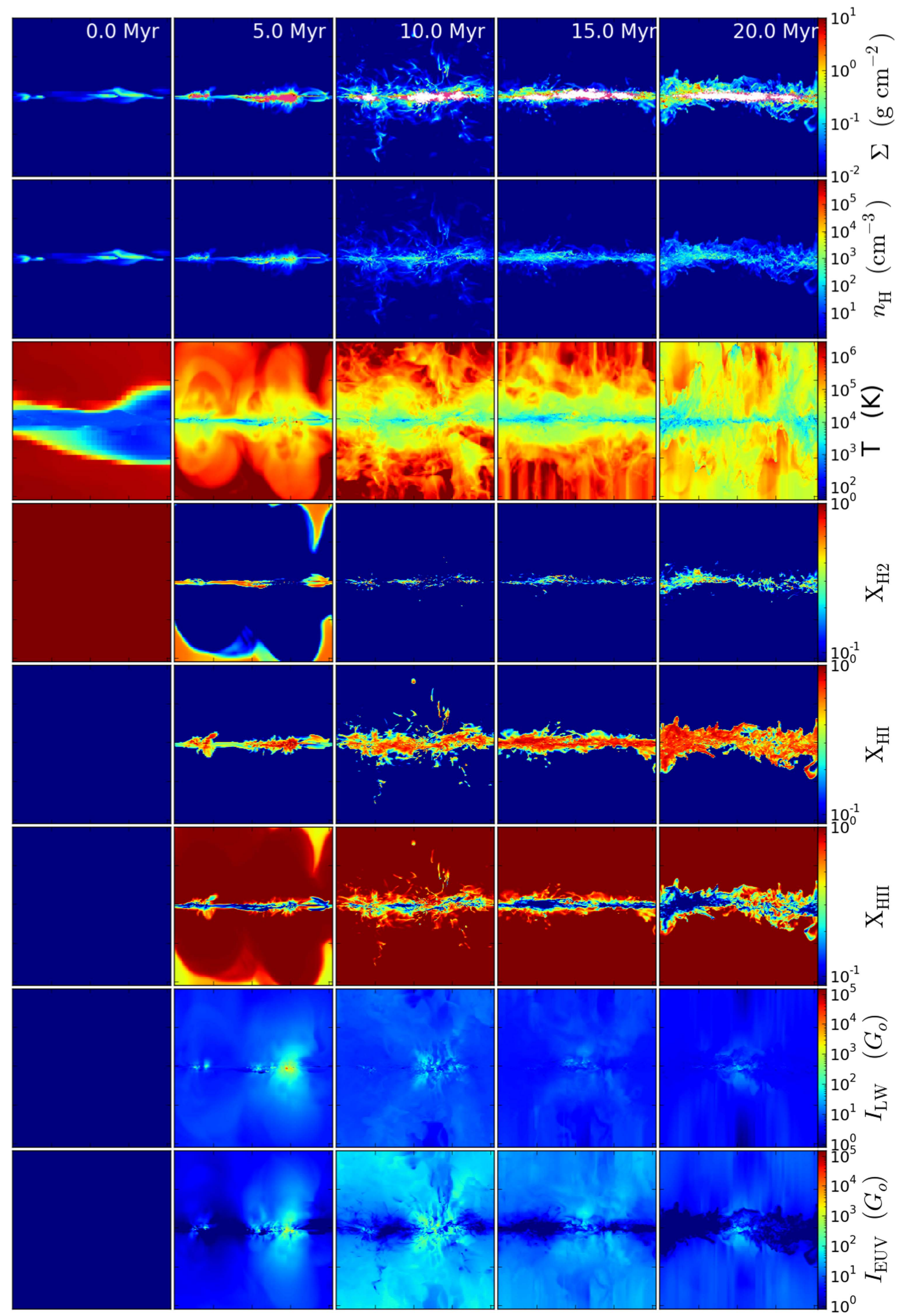

Figure 18. Same as Figure 1, but for the Supernova-only simulation (Run SN). 


\section{References}

Abel, T., Anninos, P., Zhang, Yu., \& Norman, M. L. 1997, NewA, 2, 181 Agertz, O., \& Kratsov, A. V. 2015, ApJ, 804, 18

Agertz, O., Moore, B., Stadel, J., et al. 2007, MNRAS, 380, 963

Bigiel, F., Leroy, A., Brinks, E., et al. 2008, AJ, 136, 2846

Bruzual, G., \& Charlot, S. 2003, MNRAS, 344, 1000

Bryan, G. L., Norman, M. L., O'shea, B., et al. 2014, ApJS, 211, 19

Butler, M. J., \& Tan, J. C. 2012, ApJ, 754, 5

Butler, M. J., Tan, J. C., \& Van Loo, S. 2015, ApJ, 805, 1

Chabrier, G. 2003, PASP, 115, 763

Da Rio, N., Tan, J. C., \& Jaehnig, K. 2014, ApJ, 795, 55

Dove, J. E., \& Mandy, M. E. 1986, ApJL, 311, L93

Draine, B. T., \& Bertoldi, F. 1996, ApJ, 468, 269

Eyink, G. L., Lazarian, A., \& Vishniac, E. T. 2011, ApJ, 743, 51

Falle, S. A. E. G., Hubber, D., Goodwin, S., \& Boley, A. 2012, in ASP Conf. Ser. 459, Numerical Modeling of Space Plasma Flows, ed. N. V. Pogorelov, J. A. Font, E. Audit, \& G. P. Zank (San Francisco, CA: ASP), 298

Ferland, G. J., Porter, R. L., van Hoof, P. A. M., et al. 2013, RMxAA, 49, 137

Forbes, J. C., Krumholz, M. R., Goldbaum, N. J., \& Dekel, A. 2016, Natur, 535,523

Gatto, A., Walch, S., Naab, T., et al. 2017, MNRAS, 466, 1903

Gnedin, N. Y., \& Abel, T. 2001, NewA, 6, 437

Habing, H. J. 1968, BAN, 19, 421

Hopkins, P. F., Keres, D., Murray, N., Quataert, E., \& Hernquist, L. 2012, MNRAS, 427, 968

Kennicutt, R. C. 1998, ApJ, 498, 541
Kim, C., \& Ostriker, E. 2015, ApJ, 815, 667

Kim, C., Ostriker, E. C., \& Kim, W. 2013, ApJ, 776, 1

Koda, J., Scoville, N., \& Heyer, M. 2016, ApJ, 823, 76

Krumholz, M. R., \& Matzner, C. D. 2009, ApJ, 703, 1352

Krumholz, M. R., \& Tan, J. C. 2007, ApJ, 654, 304

Lazarian, A., \& Vishniac, E. T. 1999, ApJ, 517, 700

Leroy, A., Walter, F., Brinks, E., et al. 2008, AJ, 136, 2782

Leroy, A. K., Walter, F., Sandstrom, K., et al. 2013, AJ, 146, 2

McKee, C. F., \& Ostriker, E. C. 2007, ARA\&A, 45, 565

Peters, T., Zhukovska, S., Naab, T., et al. 2017, MNRAS, 466, 3293

Rosdahl, J., Blaizot, J., Aubert, D., Stranex, T., \& Teyssier, R. 2013, MNRAS, 436, 2188

Rosdahl, J., Schaye, J., Teyssier, R., \& Agertz, O. 2015, MNRAS, 451, 34

Rosdahl, J., \& Teyssier, R. 2015, MNRAS, 449, 4380

Tan, J. C. 2010, ApJL, 710, L88

Tan, J. C., Beltran, M. T., Caselli, P., et al. 2014, in Protostars and Planets VI, ed. H. Beuther, R. S. Klessen, C. P. Dullemond, \& T. Henning (Tucson, AZ: Univ. Arizona Press), 149

Tasker, E. J., \& Tan, J. C. 2009, ApJ, 700, 358

Teyssier, R., Pontzen, A., Dubois, Y., \& Read, J. 2013, MNRAS, 429, 3068

Van Loo, S., Butler, M. J., \& Tan, J. C. 2013, ApJ, 764, 36

Van Loo, S., Tan, J. C., \& Falle, S. A. E. G. 2015, ApJL, 800, L11

Walch, S., Girichidis, P., Naab, T., et al. 2015, MNRAS, 454, 238

Wolfire, M. G., McKee, C. F., Hollenbach, D., \& Tielens, A. G. G. M. 2003, ApJ, 587, 278

Zuckerman, B., \& Evans, N. J. 1974, ApJL, 192, L149 